\title{
CYCLE ÉVOLUTIF COMPLET DE SCHISTOSOMA BOVIS
}

\section{Infection naturelle en Corse et infection expérimentale de Bullinus contortus}

\author{
Par E. BRUMPT
}

Au cours d'une des enquêtes antipaludiques que je poursuis en Corse, grâce au généreux concours de la Fondation Rockefeller, j'ai eu la bonne fortune de découvrir la cercaire encore inconnue du Schistosoma bovis (Sonsino, 1876) et de démontrer, en infectant divers mammifères, que ce parasite existait en Corse où il n'avait encore jamais été signalé.

C'est en recherchant les cercaires de mollusques divers, susceptibles d'infecter les larves d'Anopheles et aptes à jouer un rôle éventuel dans la lutte biologique contre ces redoutables vecteurs du paludisme, que j'ai trouvé la furco-cercaire qui m'a permis de faire les observations morphologiques et biologiques signalées dans ce premier mémoire.

La présence de métacercaires chez les Anopheles adultes ou larvaires est un fait connu depuis les travaux déjà aneiens de Grassi (1900), mais c'est seulement depuis les recherches de Soparkar (1917) et celles de Fülleborn (1922) que l'on connaît la morphologie de certaines cercaires capables de pénétrer dans le corps des larves et d'entraîner parfois leur mort. De mon côté, j'ai eu l'occasion d'étudier une xiphidiocercaire parasite du Planorbis corneus de la région de Gatz (Manche), susceptible de tuer expérimentalement, en quelques minutes ou en quelques heures, des larves de Stegomyia fasciata (Aedes rgypti), de Culicella morsitans, d'Aedes (Ochlerotatus) communis, d'Anopheles bifurcatus, de Theobaldia annulata et de divers autres nématocères, en particulier celles des Chironomus (1). Ces recherches seront publiées ultérieurement car jusqu’à ce jour j'ai recherché en vain le trématode adulte engendrant la forme larvaire pathogène pour les insectes énumérés ci-dessus. Si ce trématode adulte vit chez un animal domestique ou sauvage pouvant être multiplié artificiellement, ce dernier pourrait devenir indirectement un auxiliaire précieux pour la lutte contre les mous-

(1) Cette cercaire armée présente malheureusement une trop faible spécificité parasitaire, car elle s'attaque aussi, expérimentalement tout au moins, aux larves de phryganes et d'Eschna, à des larves de tabanides dont je n'ai pu obtenir l'élevage, ainsi qu'à des Asellus.

Annales de Parasitclogie, $\mathrm{T}$. VIII, $\mathrm{s}^{\circ} 1 .-1^{\mathrm{er}}$ janvier 1930 , p. 17-50. 
tiques. Il serait à ajouter à la liste déjà longue des êtres qui par leur interaction jouent des rôles divers dans la prophylaxie des maladies parasitaires.

Bien qu'il soit très difficile de se rendre compte de l'influence que les mollusques parasités par des cercaires peuvent avoir, dans la nature, sur la densité larvaire des culicidés dans le gîte où ces êtres cohabitent, et sans croire que des recherches sur ce sujet puissent conduire à préconiser une méthode prophylactique nouvelle, les mollusques d'eau douce étant des animaux trop suspects pour que leur dissémination puisse être conseillée, j'estime qu'il est bon de les entreprendre. Ces recherches peuvent en effet être poursuivies à l'occasion de l'étude des gîtes larvaires et la découverte que je viens de faire de la cercaire de Schistosoma bovis, au cours de l'empoissonnement avec des Gambusia de diverses mares de Monacia, montre qu'en dehors de leur intérêt théorique elles peuvent conduire à des mesures prophylactiques intéressant l'élevage.

La bilharziose bovine, déterminée par Schistosoma bovis, existe en Egypte où elle a été découverte en avril 1876, à Zagazig, par P. Sonsino. Depuis cette époque, elle a été signalée en Afrique méridionale (Cawston, 1920) et orientale et dans l'Ouganda (19111912), aux Indes (Bomford, 1886), dans les Etats malais, en IndoChine (Annam) (Railliet, 1899), en Sicile (Grassi et Rovelli, 1888 ; Barbagallo, 1899) et en Sardaigne (San Felice et Loi, 1897 ; Bertolini, 1908 ; Alceo, 1926).

Jusqu'à ce jour, cette infection n'avait pas été observée en France et c'est certainement par suite d'une erreur bibliographique que Khalil (1924) et Baylis (1929) disent que Schistosoma bovis existe dans le Midi de notre pays. La seule espèce de bilharzie signalée en France, Schistosoma turkestanicum (1) (Skrjabin, 1913), est très différente de l'espèce qui fait l'objet de cette communication. Ce ver a été récolté une seule fois, à l'autopsie d'une vache de la Dombes, par Marotel, en,1908; il n'a pas été signalé depuis cette époque.

Malgré la vaste répartition géographique du $S$. bovis, son évolution était en grande partie inconnue. Nous savions seulement, par les travaux de Cawston $(1920 \mathrm{~b})$, qu'un cobaye infecté au Natal avec des cercaires parasites du mollusque Physopsis africana, en octobre, avait présenté, en février, des bilharzies identifiées par R.-T. Leiper à Schistosoma bovis et que la furcocercaire pourvue d'un pharynx, désignée sous le nom de Cercaria octadena Faust

(1) Ce ver vient d'être placé dans le genre Ornithobilharzia Odhner, 1912, par E.-W. Price (1929). 
1921 (fig. 1), était encore considérée par Faust (1921) et par Cawston (1923) comme étant la forme larvaire de ce trématode.

Nos abservations faites en Corse, au laboratoire anti-paludique de Porto-Vecchio, et à mon laboratoire de la Faculté de Médecine, m'ont permis de démontrer, d'une part, que la cercaire du Schistosoma bovis est dépourvue de pharynx, comme celles des schistosomes parasites de l'homme ou d'autres mammifères dont l'évolution est connue, et, d'autre part, que l'hòte intermédiaire de ce ver, en Corse, est le Bullinus contortus (1), hôte habituel de Schistosoma hæmatobium dans le bassin méditerranéen, en Egypte, et dans le proche Orient.

Infection des Mollusques. - J'ai rencontré les mollusques infectés dans un certain nombre de mares (fig. 2 et 3 ) du village de Monacia situé à l'ouest de Bonifacio, au cours de deux expéditions effectuées le 15 et le 20 août 1929. La proportion de mollusques émettant des furcocercaires de Schistosoma bovis était d'environ 1 pour cent à cette époque de l'année, sur 2.000 exemplaires examinés vivants, par lots, dans des tubes exposés au soleil.

Le pourcentage de mollusques infectés est plus faible si, au lieu de l'établir d'après les mollusques récoltés dans les mares à Schistosoma bovis, on le calcule sur le total de tous les animaux récoltés dans les diverses mares de la localité. Nous trouvons alors que (v. p. 22) sur environ 6.000 mollusques, examinés par lots d'importance diverse, il y en avait au moins 29 parasités, soit environ 0,5 pour cent. Ce chiffre de 29 est cependant un minimum, puisque nous considérons comme renfermant un bullin parasité les tubes où des furcocercaires étaient observées, mais dont les mollusques n'ont pas été ultérieurement isolés faute de temps. Il est d'ailleurs probable, étant données les périodes négatives

(1) Dans les pays où la bilharziose urinaire humaine est due à la présence de mollusques du genre Bullinus, les auteurs attribuent généralement les furcocercaires observées au Schistosoma hamatobium. Le présent travail montre que celles ci peuvent appartenir également au cycle du $S$. bovis, parasite qui sera certainement rencontré dans les pays de l'Afrique mineure où les mollusques hôtes intermédiaires sont très abondants. La présence du Schistosoma bovis aux Indes et en Extrême-Orient, dans des régions où ni Bullinus contortus, ni Physopsis africana ne se rencontrent, montre qu'il existe encore d'autres hôtes intermédiaires susceptibles d'assurer le cycle évolutif de ce parasite. 
d'évacuation des cercaires par des bullins certainement parasités, que par la dissection des animaux le pourcentage aurait été sensi-

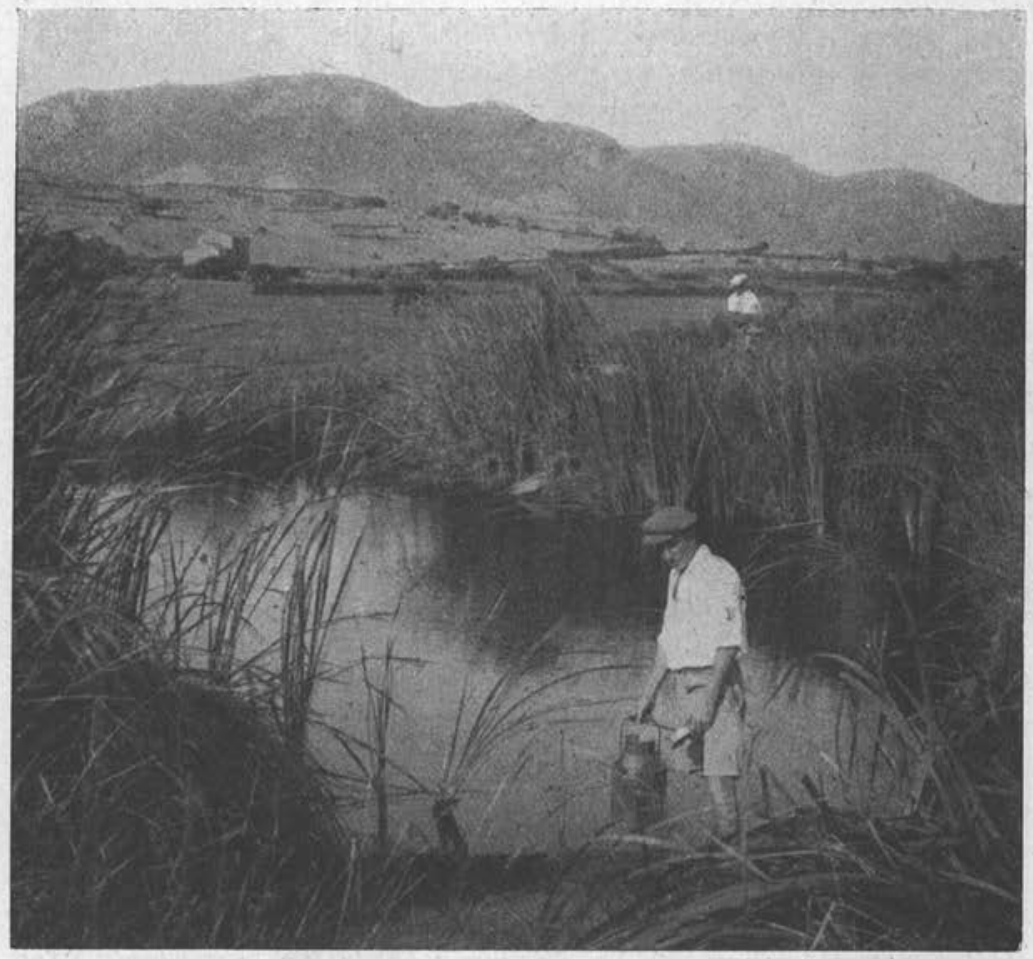

Fig. 2. - Une mare de la région de Monacia (Corse) photographiée le 15 août 1929. Au premier plan, à l'endroit où se trouve une personne tenant un bidon rempli de Gambusia et où le bétail vient boire, on peut récolter sur la vase de 10 à 30 Bullinus contortus par décimètre carré. Environ 1 pour cent de ces mollusques émettaient des furcocercaires de Schistosoma bovis. Au second plan, une personne se trouve au-dessus d'un fossé $(40 \mathrm{XI})$ abrité par une végétation touffue, à sec depuis au moins quinze jours, dans lequel se trouvaient de nombreux Bullinus encore vivants.

blement relevé, moins cependant que dans le cas du Paramphistomum cervi chez le même hôte.

Les plus petits mollusques infectés mesuraient quatre millimètres (1) de hauteur, les plus gros dix millimètres.

(1) C'est par erreur que, dans ma note préliminaire présentée à l'Académie des Sciences (séance du 12 novembre 1929), par le professeur Leclainche, j’ai écrit cinq millimètres. Dans deux cas les mollusques parasités avaient, au maximum, quatre millimètres et peut-être moins car leur isolement du lot dans lequel ils se trouvaient n'a pas été fait faute de temps. 
La récolte des Bullinus, très facile à effectuer avec un filet dans certains gîtes où ils se trouvent sur des characées ou d'autres plantes fixées sur le fond des mares, est beaucoup plus difficile dans d'autres où ils sont fixés sur les pierres ou sur les tiges de certaines plantes. Dans ces derniers cas, il faut, ou les récolter un à un sur

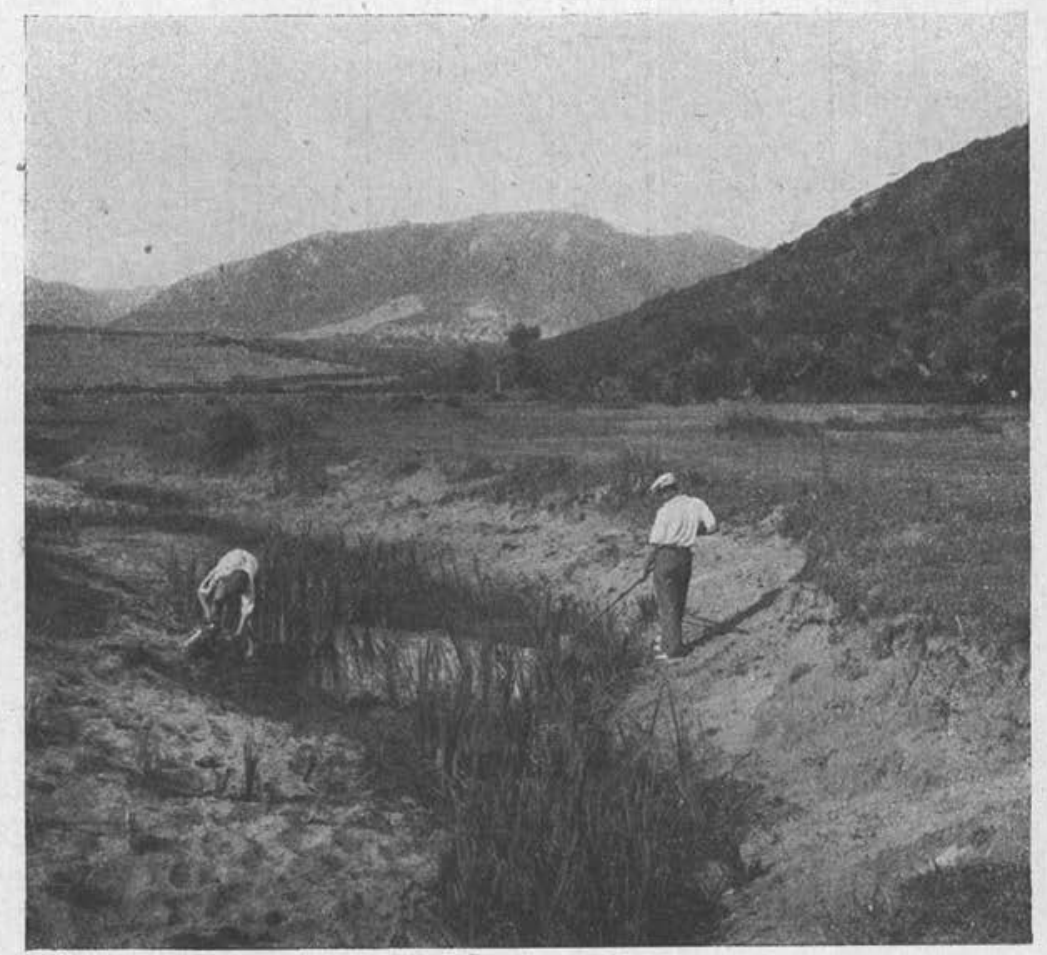

FIg. 3. - Autre mare-abreuvoir de Monacia où les Bullinus contortus infectés de furcocercaires dans le même pourcentage que dans la mare de la fig. 2, étaient extrêmement nombreux sur des characées.

les grosses pierres ou brosser de petites pierres dans un récipient quelconque, ou encore, comme le montre la photographie ci-jointe, arracher de grandes touffes de Typha ou de Sparganium et, après avoir,ôté une partie de la vase qui les recouvre, les laver dans des récipients appropriés (fig. 4 ).

On peut, par ces procédés, récolter en divers points de Corse, dans la région de Campo dell Oro, aux environs d'Ajaccio, et dans celle de Monacia, des milliers de Bullinus. Pour donner une idée de 
Répartition et fréquence de la cercaire de Schistosoma bovis en Corse

\begin{tabular}{|c|c|c|c|c|c|c|}
\hline Localité & DATE & Gites & $\begin{array}{c}\text { Nombre } \\
\text { D'EXEMPLAIRES }\end{array}$ & $\begin{array}{l}\text { DIMENSIONS } \\
\text { EN } \\
\text { MILLIMÈTRES }\end{array}$ & 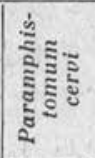 & 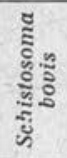 \\
\hline 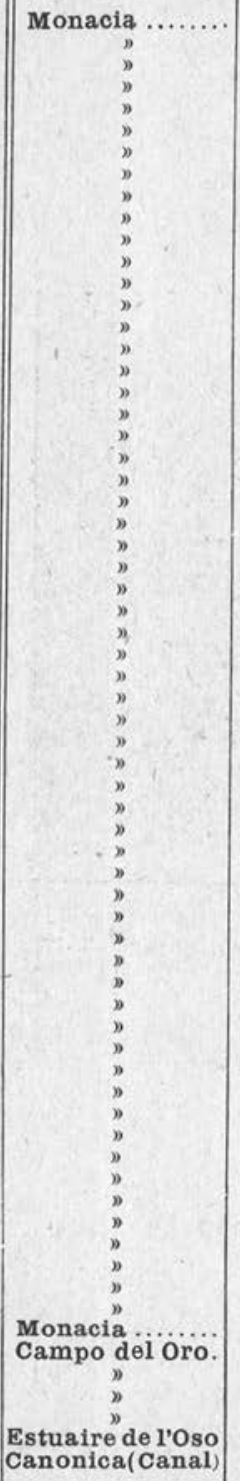 & 15 et 20-8-1929 & $\left|\begin{array}{cccc}40 & \mathrm{XI} \\
31 & c & \mathrm{XI} \\
& b & b \\
31 & b & \mathrm{XI} \\
31 & d e & \mathrm{XI} \\
34 & \mathrm{XI} \\
36 & \mathrm{XI}\end{array}\right|$ & $\begin{array}{r}95 \\
250 \\
50 \\
100 \\
100 \\
100 \\
100 \\
100 \\
100 \\
300 \\
120 \\
42 \\
30 \\
24 \\
5 \\
6 \\
6 \\
5 \\
5 \\
8 \\
5 \\
10 \\
6 \\
6 \\
5 \\
10 \\
150 \\
150 \\
150 \\
150 \\
150 \\
150 \\
5 \\
10 \\
7 \\
5 \\
5 \\
60 \\
12 \\
10 \\
6 \\
6 \\
7 \\
12 \\
20 \\
15 \\
15 \\
50 \\
10 \\
400 \\
400 \\
400 \\
400 \\
10 \\
10 \\
20 \\
30 \\
30 \\
30 \\
100 \\
1000 \\
60 \\
58 \\
25 \\
60 \\
140 \\
500 \\
\text { a } \\
50 \\
\end{array}$ & 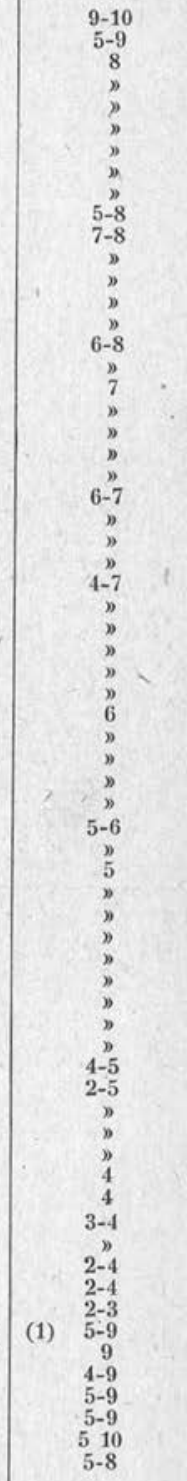 & 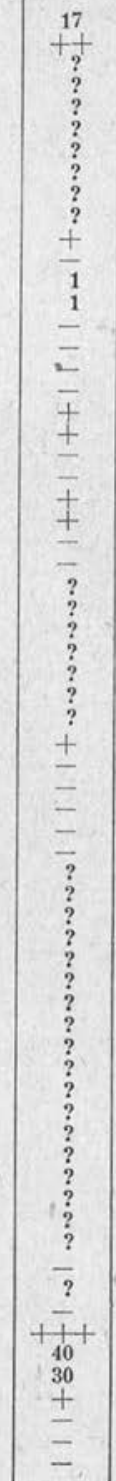 & 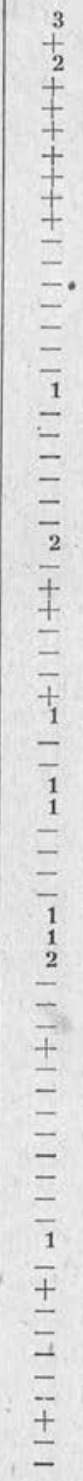 \\
\hline
\end{tabular}

(1) Ces exemplaires étaient dans un diverticule de la mare 39 (XI), à sec depuis au moins quinze jours mais abrité par de la végétation. Mis dans l'eau, presque tous ont montré une activité identique à celle de ceux pris dans l'eau de la mare. 
leur abondance, je dirai qu'en certains endroits on peuí en trouver de 10 à 100 au décimètre carré, beaucoup plus qu'en certains points du Maroc, à Marrakech en particulier, où la bilharziose humaine est répandue et où j'ai eu l'occasion d'étádier la distribution et la biologie de ces mollusques en 1923.

Pour savoir, sur le terrain, quelles étaient les mares renfermant des Bullinus contortus infectés, afin de ne pas perdre de temps à

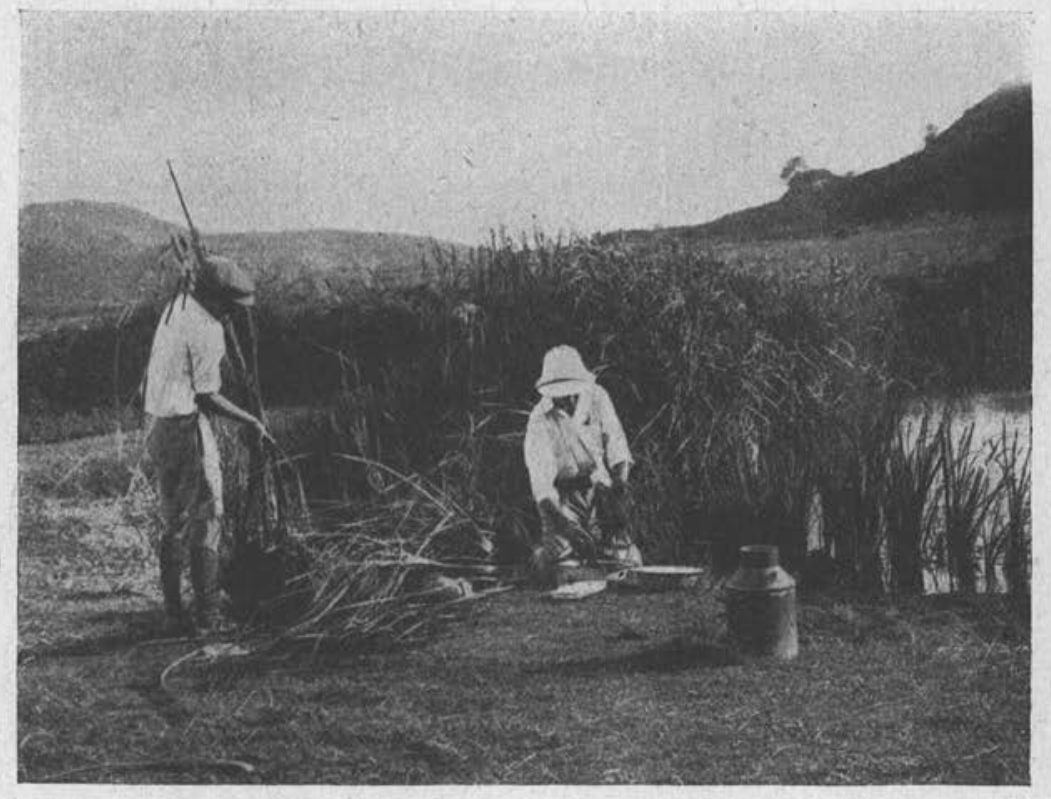

Fı́. 4. - Pour récolter rapidement de grands exemplaires de Bullinns contortus le mieux est d'arracher de grosses touffes de Typha et de laver leurs parties immergées dans des récipients divers.

récolter de nombreux exemplaires négatifs, des sondages d'épreuve étaient rapidement effectués dans les points suspects et 200 ou 300 mollusques étaient placés au soleil dans un tube Borrel. En examinant les tubes à la loupe (fig. 5), il était parfois facile de rencontrer des furcocercaires au bout de quelques minutes. Quand cet examen était positif, un grand nombre de mollusques étaient alors récoltés et emportés au laboratoire de PortoVecchio, où un triage pouvait être effectué en isolant un à un les gros exemplaires de Bullinus et en mettant les autres par lots pour faciliter l'isolement des sujets parasités. La photographie (fig. 6) 
montre, sur les marches de la porte du laboratoire de PortoVecchio, en 4 et 5 des tubes d'isolement et en 1 et 3 des cristallisoirs renfermant de l'eau chargée de cercaires de $S$. bovis dans laquelle haignent des hérissons de Corse aimablement procurés par le Docteur G. Coulon, médecin résident à Porto-Vecchio.

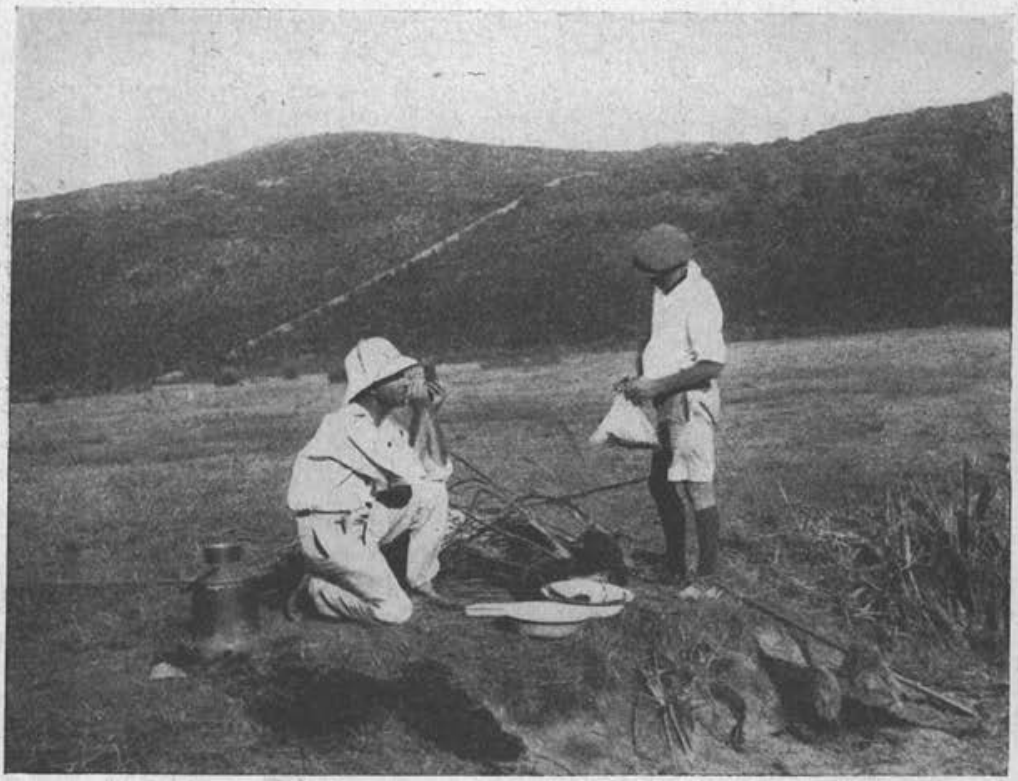

Fı́. 5. - Pour éviter de perdre du temps à récolter des Bullinus dans des mares où ils né sont pas infectés, le mieux est de faire dans tous les gîtes un sondage rapide permettant de capturer de 200 à 300 exemplaires que l'on place dans un tube Borrel exposé au soleil. En attendant quelques minutes, il est facile d'observer des furcocercaires à la loupe et même à l'œil nu, quand elles sont nombreuses. Si l'épreuve est positive, d'autres mollusques sont récoltés et mis avec des plántes aquatiques humides, dans un sac de toile numéroté.

Infestations expérimentales. - En partant de ces cercaires, j'ai pu infecter à Porto-Vecchio, du 20 au 25 août, un hérisson (Erinaceus europaeus) qui a succombé 37 jours après le premier bain dans l'eau chargée de furcocercaires, avec des embolies de vers, accouplés ou non, dans les vaisseaux du foie, du pancréas et du mésentère (fig. 7 et 8).

De retour à Paris, j’ai pu infecter des souris blanches, à partir du 30 août, avec les mollusques apportés de Corse. L'une d'elles (127 XI), sacrifiée le $35^{\circ}$ jour, montrait dans le foie et les veines du 
mésentère de très jeunes vers des deux sexes ; une autre $(140 \mathrm{XI})$, sacrifiée le $63^{\circ}$ jour, hébergeait quatorze mâles et quatorze femelles dont douze accouplés. Plusieurs femelles présentaient des œufs non mûrs dans leur utérus et on en rencontrait de non mûrs également dans la paroi du rectum et dans le parenchyme hépatique qui, chez le bœuf (1), est le lieu d'élection où les œufs se trouvent le plus

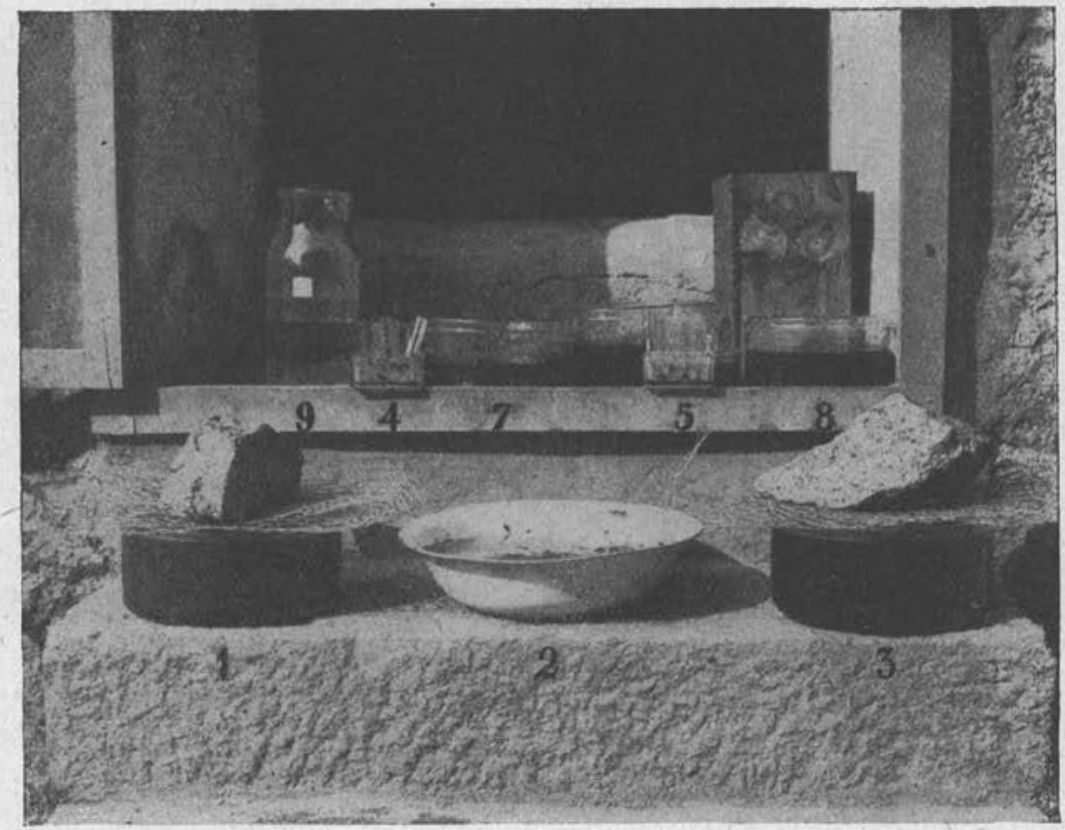

FIG. 6. - Isolement individuel des mollusques des lots positifs, au laboratoire de Porto-Vecchio, dans les tubes groupés en 4 et en 5 . Les cristallisoirs 1 et 3 renferment chacun un hérisson et de l'eau chargée de cercaires de Schistosoma bovis. Les autres récipients renferment des Bullinus de divers gîtes.

facilement à l'examen microscopique, ainsi qu'il résulte de mes recherches effectuées à la Faculté de Médecine du Caire, au laboratoire de mon collègue $\mathrm{M}$. Khalil.

Les vers màles et femelles adultes trouvés chez la souris sont plus petits que ceux observés chez le bœuf, mais ils présentent la même structure anatomique, bien décrite par M. Khalil en 1924.

(1) Je suis heureux d'avoir l'occasion de remercier ici le directeur de l'abattoir du Caire, M. Ibrahim Effendi Magieb et les vétérinaires, MM. Husni et Wahba Michhail qui ont facilité mes recherches et m'ont fait profiter de leur grande expérience. 
Le temps m'a manqué, en Corse, pour mettre en évidence les Schistosoma bovis dans les vaisseaux veineux des bovins ou des ovins et je n'ai pas trouvé d'œufs de ce ver dans les déjections d'une vache (fig. 15) et d'un veau (fig. 16), atteints de cachexie aqueuse avec œedème intermaxillaire (" bouteille ") très prononcé, et d'un veau sain s'abreuvant et se baignant dans les mares de Monacia.

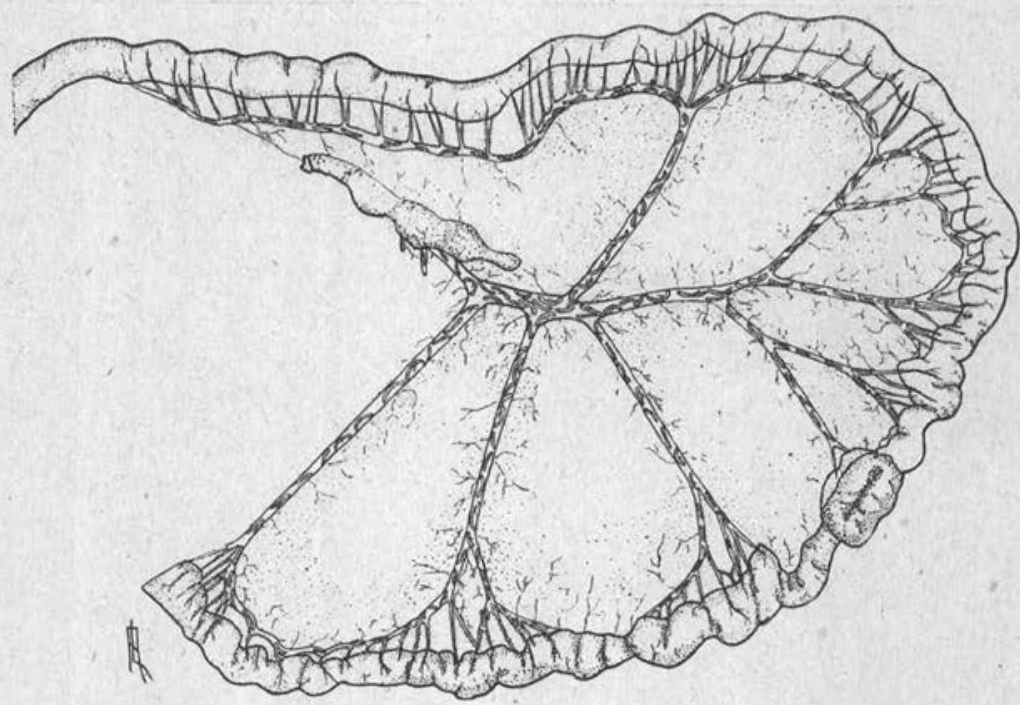

Fig. 7. - Intestin grêle du hérisson 61 XI, mort le 37e jour. Les veines du mésentère sont remplies de vers des deux sexes déjà accouplés. Les mâles mesurent environ 3 millimètres et les femelles 4 millimètres de longueur. Il est exceptionnel de rencontrer des vers dans les veinules situées au bord de l'intestin. Il n'a pas été possible d'assister à la ponte, même dans des cas d'infection intense chez la souris, où les femelles adultes étaient nombreuses.

Ce fait n'a rien de surprenant, car je n'ai pas trouvé davantage d'œufs en Egypte, dans les déjections d'un bœuf soudanais ayant de nombreux vers dans le mésentère. Par contre, j'ai vu dans les selles dés trois bovidés corses signalés ci-dessus des œufs, parfois très nombreux, du Paramphistomum cervi, trématode très commun à l'état larvaire chez le Bullinus contortus.

Comme j'espère étudier prochainement la morphologie du Schistosoma bovis aux divers stades de son existence chez ses hôtes définitifs expérimentaux et chez son hôte intermédiaire, je me contenterai de résumer, dans les lignes qui suivent, quelques-unes des observations que j'ai pu faire. 
Comme je l'ai dit plus haut, les schistosomes adultes, récoltés chez le cobaye et chez les souris, sont plus petits que ceux récoltés chez le bœuf.

En ce qui concerne les màles, mes observations, sur un certain nombre d'exemplaires récoltés à l'abattoir du Caire, concordent avec celles de divers auteurs (Sonsino, San Felice et Loi, Barbagello, Bertolini, Khalil), leur longueur varie entre 9 et 20 millimètres. Expérimentalement chez le cobaye (117 XI), des spécimens âgés de

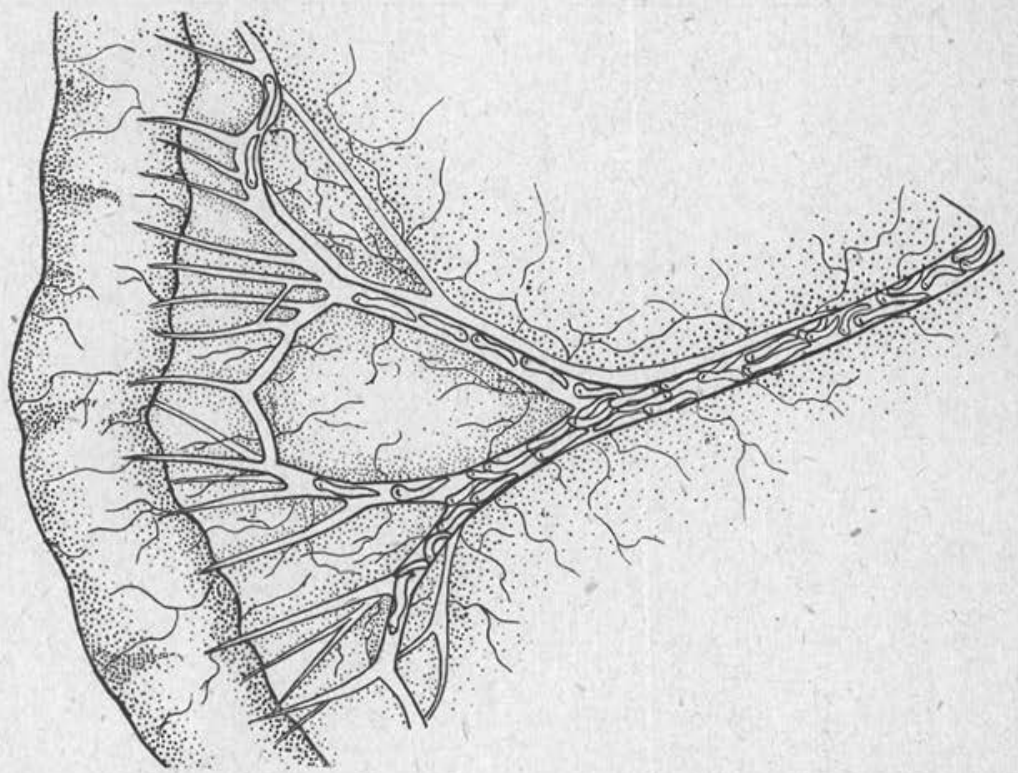

Fig. 8. - Portion de la fig. 7 agrandie pour montrer l'absence de schistosomes, habituelle, tout au moins dans les infections récentes, dans les yeinules bordant l'intestin.

77 à 81 jours mesuraient de 8 à 10 millimètres, mais il existait, comme toujours dans les infections à schistosomes, des exemplaires plus petits mal développés. Chez la souris (150 XI), des mâles âgés de 84 à 87 jours mesuraient respectivement : 4,$5 ; 5,2 ; 4,75 ; 4,25$; 6,$5 ; 4,5 ; 6,5$ millimètres. Chez une autre souris, un mâle, âgé de 95 jours, tué par l'eau chaude, mesurait 12 millimètres.

Les femelles provenant du bœuf mesurent de 20 à 27 millimètres (Bertolini, 1908) et de 12 à $17 \mathrm{~mm}$. (M. Khalil, 1924). J'ai trouvé, pour quatre femelles entières récoltées chez un bœuf soudanais, sacrifié au Caire, les dimensions suivantes : $12 ; 14 ; 12$ et 13,25 millimètres. Ces quatre femelles étaient adultes et renfermaient 
des œufs dans leur utérus. Chez la souris (150 XI), sept exemplaires âgés de 84 à 87 jours mesuraient : $6 ; 6 ; 5,40 ; 6,75 ; 7,15 ; 7,2$ et 7,5 millimètres. Chez d'autres souris, des exemplaires de $8,9,10$, 12,25 et 16,1 millimètres ont été observés.

CEufs. - La femelle de $S$. bovis, comme celle des autres schistosomes dont l'étude a été faite, pond ses œufs à un stade peu développé. L'oocyte, clair et réfringent, se détache sur la masse opaque

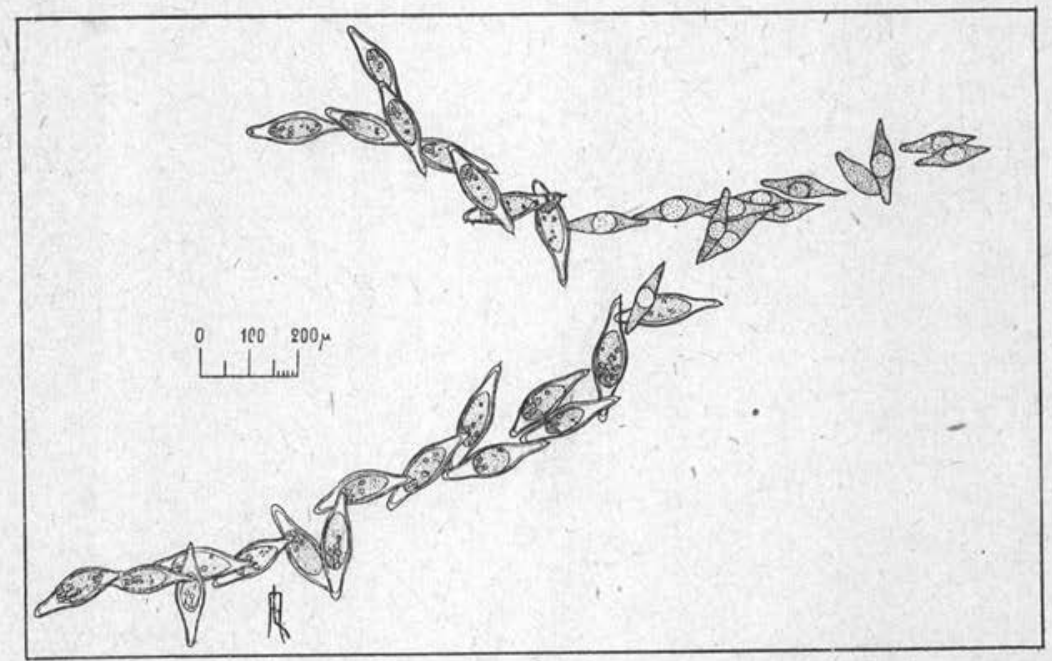

Fıg. 9. - Eufs de Schislosoma bovis déposés dans une veinule de la paroi intestinale d'une souris (142 XI) morte le $73^{\circ}$ jour de son infection. Les œufs qui se trouvent à droite dans le tronc veineux sont jeunes et montrent au centre un espace clair constitué par l'oocyte segmenté. Les œufs qui remplissent les deux divisions de la veinule ont été pondus depuis quelques jours, ils ont grandi et renferment un miracidium bien développé. Ces mêmes faits s'ohservent dans les parois intestinales de souris infectées par $S$. mansoni et $S$. hæmatobium.

des cellules vitellines. Ces dernières, autant qu'il est possible de les compter, car elles se trouvent parfois sur deux ou trois plans, sont au nombre de 30 à 40 . Les œufs, qui n'évoluent pas durant leur rapide traversée de l'utérus, se trouvent en nombre variable dans cet organe. Des femelles âgées de 84 à 87 jours renfermaient respectivement $38,16,20,4,3,39$ œufs ; d'autres, de 86 à 89 jours, présentaient $10,7,3,9,13.7,5,11$ et 5 œufs.

Dans la plupart des ouvrages, on trouve, en ce qui concerne les dimensions de l'œuf de $S$. bovis, celles indiquées par Sonsino se rapportant sans aucun doute à des œufs utérins. Les dimensions de 
160 à $180 \mu$ de long sur 40 à $50 \mu$ de large sont données par Sonsino, Cobbold, Leuckart, Barbagello, Khalil, Baylis. Le seul auteur, à ma connaissance tout au moins, qui ait donné des mensurations d'œufs trouvés dans les tissus de l'hôte, est Bertolini qui indique pour les œufs utérins les dimensions de 132 à $165 \mu$ sur 33 à $44 \mu$ et pour ceux

\begin{tabular}{|c|c|c|c|}
\hline $\mathrm{N} \cdot$ DES SOURIS & N* DEs $Q$ & $\begin{array}{l}\text { AGE DES } q \\
\text { (EN JOUns) }\end{array}$ & DIMENSIONS DES CEUFS \\
\hline $\begin{array}{ll}140 & \text { XI......... } \\
144 & \text { XI...... } \\
146 & \text { XI....... } \\
150 & \text { XI........ }\end{array}$ & $\begin{array}{r}1 \\
1 \\
1 \\
2 \\
3 \\
4 \\
5 \\
6 \\
7 \\
\text { H. S. } \\
1 \\
2 \\
\\
3 \\
4 \\
5 \\
6 \\
7 \\
8 \\
9 \\
10 \\
11 \\
12 \\
13\end{array}$ & 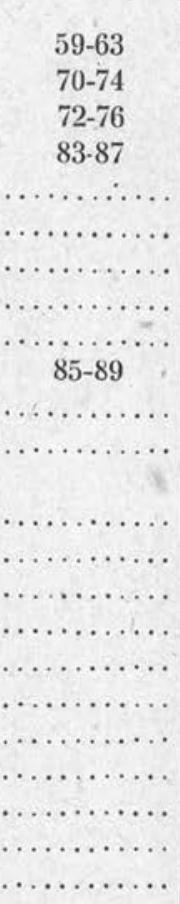 & $\begin{array}{l}175 / 42,160 / 40,175 / 40 \\
170 / 45 \\
145 / 45,150 / 50,175 / 50 \\
140 / 50,160 / 50,175 / 40 \\
160 / 45,170 / 45,175 / 40 \\
135 / 40,135 / 40 . \\
129 / 40,130 / 35,140 / 30,140 / 30 \\
165 / 40,170 / 40 \\
165 / 40,160 / 35 \\
165 / 50,170 / 50,165 / 50 \\
115 / 38,115 / 40,125 / 35 \\
160 / 42,150 / 42,175 / 38,160 / 40 \\
160 / 35,150 / 35 \\
110 / 40,110 / 40,115 / 40 \\
150 / 47,157 / 40,150 / 47 \\
120 / 40,120 / 45 \\
140 / 46,150 / 50,160 / 50,165 / 50 \\
109 / 32,125 / 32,130 / 32 \\
125 / 40 . \\
130 / 40,138 / 35,138 / 38 \\
150 / 38,150 / 45,155 / 55 \\
175 / 45,175 / 50 \\
63 / 20,110 / 30 \\
120 / 50,130 / 50,140 / 55,150 / 56\end{array}$ \\
\hline
\end{tabular}

trouvés dans les tissus du bœuf 181 à 198 sur 39 à 49 ; certains œufs observés par lui mesuraient même 214 à 247 avec une largeur variant de 53 à $59 \mu$.

De mon côté, sur des coupes de foie d'un bœuf soudanais, j'ai rencontré des œufs relativement peu déformés mesurant de 160 à $180 \mu$ de long sur 40 à 50 de large. Sur plusieurs femelles provenant d'un bœuf soudanais, j'ai trouvé pour les œufs utérins les dimensions suivantes : $140 / 55 ; 160 / 50 ; 160 / 50 ; 170 / 35 ; 170 / 50$; 
$170 / 50 ; 180 / 50 ; 180 / 50 ; 180 / 50 ; 185 / 40 ; 192 / 41 ; 205 / 40$. Chez une femelle donnée, les œufs ont sensiblement la même largeur mais leur longueur varie dans une certaine limite. Chez les femelles obtenues expérimentalement, les œufs utérins sont en général un peu plus petits que ceux récoltés chez le bœuf. C'est ainsi que chez le cobaye $(177 \mathrm{XI})$ une femelle, âgée de 77 à 81 jours, avait des œufs de $100 / 46 ; 110 / 40 ; 130 / 41$ et $169 / 45 \mu$.

Chez diverses souris, j'ai relevé les chiffres donnés dans le tableau ci-dessus (p. 29).

Les œufs, observés dans le produit de raclage de l'intestin, présentent des dimensions identiques à celles des œufs utérins quand ils viennent d'être déposés et de plus grandes dimensions quand le miracidium est formé. D'après les observations faites sur des souris mortes ou sacrifiées peu de temps après l'apparition des premiers œufs dans les tissus, j'estime que le miracidium peut être entièrement formé deux à trois jours après la ponte. Pour établir ce point, j'ai essayé en vain de faire évoluer les œufs jeunes en conservant des fragments d'intestin très parasités dans des flacons renfermant une solution d'acide chromique à 1 pour 2.000. Peut-être serait-il plus facile de résoudre ce problème en plaçant un fragment aseptique de foie ou de rate renfermant de nombreux œufs jeunes dans du plasma sanguin à la température de $37^{\circ} \mathrm{C}$.

En raclant l'intestin d'une souris $(151 \mathrm{XI})$, morte le $89^{\circ}$ jour après la première infection et 85 jours après la dernière, j'ai observé des œufs ayant les dimensions suivantes :

a. - Eufs non mûrs en voie d'évolution normale : 120/50, $160 / 40,170 / 55,175 / 45,180 / 60$.

b. - QEufs mûrs avec miracidium bien développé : 230/70, $235 / 80,260 / 80,270 / 80,280 / 90$.

Enfin, dans les déjections d'une souris (146 XI), morte 76 jours après la première infection et 72 jours après la dernière, les dimensions relevées pour les œufs sont données dans le tableau de la page 31.

Comme on le voit, les œufs atteignent dans les déjections de la souris, dans lesquelles il est exceptionnel de trouver des œufs dégenérés, des dimensions aussi grandes et même plus grandes que celles signalées par Bertolini dans la paroi intestinale du bœuf.

Vers adultes. - Dans les infections expérimentales, les vers adultes se rencontrent dans les vaisseaux veineux du foie, dans la veine splénique (fig. $4, \mathrm{pl} .1$ ), dans les veines de l'estomac et dans celles du mésentère. Une seule fois, sur 19 autopsies, des vers adultes au nombre de trois ont été trouvés dans un fragment de poumon. Les tableaux des pages 32 et 33 , donnant un résumé des autopsies 


\begin{tabular}{|c|c|c|}
\hline $\mathrm{N} \cdot$ Des aers & DiMENSIONS DES GUFS & DiMENSIONS DES MIRACIDIUM \\
\hline $1 \ldots \ldots \ldots \ldots \ldots$ & $190 / 60$ & $\forall$ \\
\hline $2 \ldots \ldots \ldots \ldots \ldots \ldots$ & $200 / 60$ & $120 / 40$ \\
\hline $3 \ldots \ldots \ldots \ldots \ldots \ldots$ & $205 / 60$ & $100 / 40$ \\
\hline $4 \ldots \ldots \ldots \ldots \ldots \ldots$ & $200 / 60$ & $120 / 40$ \\
\hline $5 \ldots \ldots \ldots \ldots \ldots \ldots$ & $23 / 80$ & $140 / 55$ \\
\hline $6 \ldots \ldots \ldots \ldots \ldots$ & $210 / 80$ & $150 / 70$ \\
\hline $7 \ldots \ldots \ldots \ldots \ldots$ & $210 / 60$ & $125 / 45$ \\
\hline $8 \ldots \ldots \ldots \ldots \ldots$ & $225 / 65$ & $125 / 50$ \\
\hline $9 \ldots \ldots \ldots \ldots \ldots \ldots$ & $230 / 65$ & $130 / 50$ \\
\hline $10 \ldots \ldots \ldots \ldots \ldots \ldots$ & $215 / 60$ & $135 / 50$ \\
\hline $11 \ldots \ldots \ldots \ldots \ldots$ & $210 / 60$ & $125 / 45$ \\
\hline $12 \ldots \ldots \ldots \ldots \ldots \ldots$ & $210 / 55$ & $120 / 45$ \\
\hline $13 \ldots \ldots \ldots \ldots \ldots \ldots$ & $230 / 65$ & $130 / 50$ \\
\hline $14 \ldots \ldots \ldots \ldots \ldots \ldots$ & $220 / 65$ & $125 / 45$ \\
\hline $15 \ldots \ldots \ldots \ldots \ldots$ & $200 / 60$ & $120 / 40$ \\
\hline $16 \ldots \ldots \ldots \ldots \ldots \ldots$ & $200 / 60$ & $130 / 40$ \\
\hline
\end{tabular}

effectuées, montrent que le foie est toujours plus infecté que l'ensemble des autres organes de la cavité abdominale placés sous la rubrique mésentère, mais comprenant également les vers rencontrés dans des vaisseaux autres que ceux de ce viscère. Cependant, chez deux souris contaminées par les cercaires d'un seul mollusque et présentant des infections par des vers du sexe mâle, la répartition des schistosomes était différente. C'est ainsi que chez la souris 197 XI, sacrifiée étant bien portante le $89^{\circ}$ jour après le début de l'expérience, le foie ne renfermait que 17 exemplaires tandis que le mésentère en hébergeait 74 .

Chez une seconde souris (194 XI), sacrifiée étant également bien portante le $113^{\circ}$ jour après l'infection par un Bullinus contortus différent de celui utilisé pour la souris 197 XI, le mésentère renfermait 210 mâles et le foie, d'où le grand lobe avait été séparé, 78 seulement. Comme le grand lobe représente environ le tiers du foie, on peut admettre que ce dernier organe renfermait environ 120 mâleś. Est-ce l'absence de femelles' quí détermine cet habitat particulier des vers mâles? C'est ce que les expériences en cours me permettront peut-être d'établir.

Le nombre des vers rencontrés est très variable suivant les animaux. Le hérisson $61 \mathrm{XI}$, infecté en Corse et mort de son infection le $37^{\circ}$ jour, renfermait certainement plus de 2.000 vers dans le foie (fig. 3 , pl. 1) et le mésentère. Le cobaye $177 \mathrm{XI}$, sacrifié très bien portant le $81^{\circ}$ jour après le début de l'infection, avait 737 adultes 


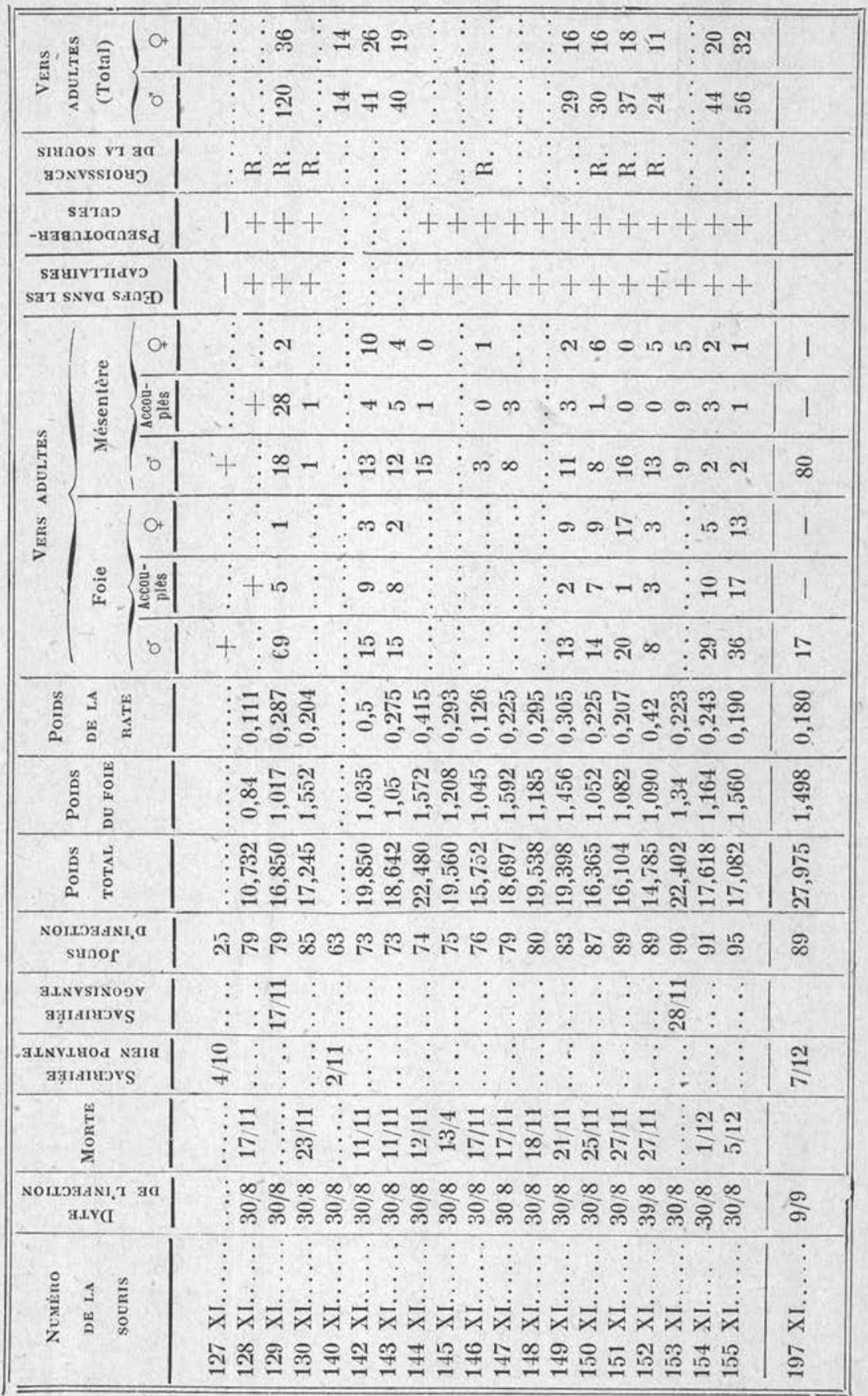




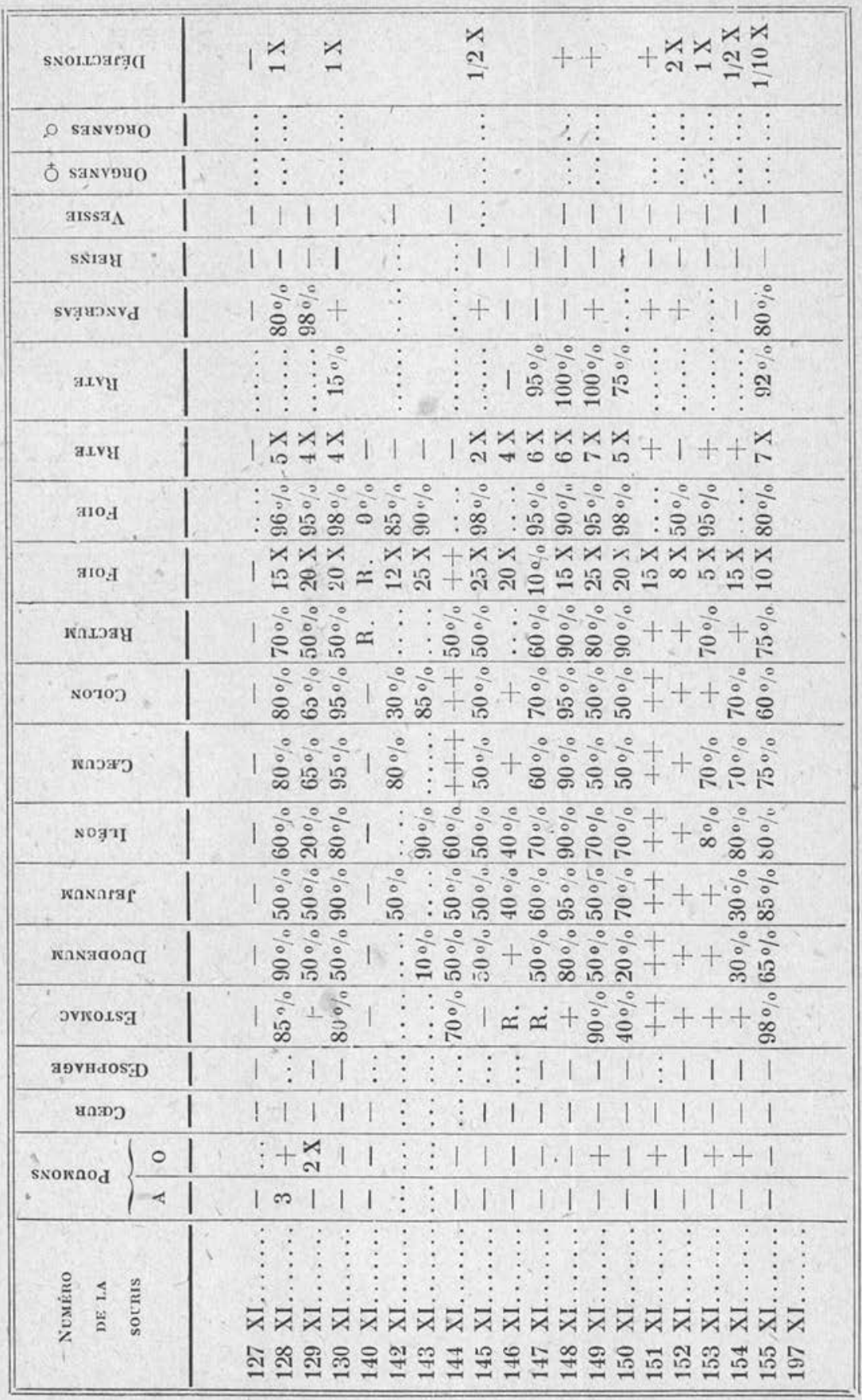

AnNales de Parasitologie, T. VIII, $\mathrm{N}^{\circ} 1 .-1^{\mathrm{er}}$ janvier 1930. 
mâles et seulement 3 femelles dans ses organes, ce qui tient à des causes que nous étudierons dans un travail ultérieur, traitant des infections expérimentales à un seul sexe que j'ai observées chez des souris infectées par $S$. hæmatobium et par $S$. bovis, mais que je n'ai pas encore réussi à obtenir avec $S$. mansoni.

Chez les souris, ainsi qu'on pourra s'en rendre compte sur le tableau d'ensemble de la page 32 , des exemplaires d'une taille relativement faible peuvent supporter une infection intense. La souris $129 \mathrm{XI}$, sacrifiée agonisante 79 jours après le début de l'infection et environ 19 jours après le début de la ponte des femelles, renfermait 120 mâles et 36 femelles. La souris 155 XI, morte le $95^{\circ}$ jour, hébergeait un total de 56 mâles et de 32 femelles.

Dans les infections expérimentales, les schistosomes mâles sont plus nombreux que les femelles. C'est ainsi que chez les dix souris (tableau p. 32), pour lesquelles tous les schistosomes adultes ont été récoltés, on compte 435 mâles pour 169 femelles. En admettant même que quelques-unes de ces dernières aient échappé aux investigations, le nombre de mâles est supérieur au double du nombre des femelles.

Les femelles de $S$. bovis, chez la souris (1), deviennent adultes beaucoup plus tard que celles de $S$. mansoni, d'après mes observations tout au moins. En effet, c'est chez l'exemplaire $140 \mathrm{XI}$, sacrifié le $63^{\circ}$ jour, qué j'ai trouvé les premiers œufs, non mûrs d'ailleurs, déposés dans certains organes, tandis que dans les infections à $S$. mansoni les souris présentent déjà des œufs avec miracidium, dans le foie, dès le $36^{\circ}$ jour (souris $113 \mathrm{XI}$ ) et peut-être même un peu plus tôt.

Mes expériences sont de date trop récente pour me permettre de dire quelle est la longévité des vers chez les souris qui sont des hôtes anormaux chez lesquels ils doivent succomber après quelques mois, comme je l'ai observé dans le cas de $S$. hæmatobium et de $S$. mansoni chez ces mêmes animaux.

Fécondité des femelles de $S$. bovis. - Je ne crois pas qu'aucun auteur ait tenté d'apprécier la fécondité des femelles de schistosomes, comme cela a été établi pour certains helminthes intestinaux. L'examen de femelles adultes, en particulier celles qui ne renferment qu'un ouf, rarement deux ou trois, dans leur utérus, comme celle du Schistosoma mansoni, permet d'admettre avec quelque raison que ces femelles sont très peu prolifiques ; c'est l'opinion que

(1) Chez un hérisson (61 XI) mort le 37 e jour de son, infection, les vaisseaux mésentérique et hépatiques étaient remplis de vers déjá accouplés. L'évolution s'effectue peut-être plus rapidement chez cet animal sur lequel je poursuis de nouvelles études. 
j'ai émise dans mon Précis de parasitologie (1922, p. 375 et p. 378). C'est également l'opinion formulée par Faust et Meleney (1924, p. 182) dans leur belle monographie du Schistosoma japonicum. Ces auteurs attribuent la gravité des lésions, observées dans les infections expérimentales par ce dernier parasite, à la grande fécondité des femelles qui peuvent présenter, âgées seulement de 33 jours,

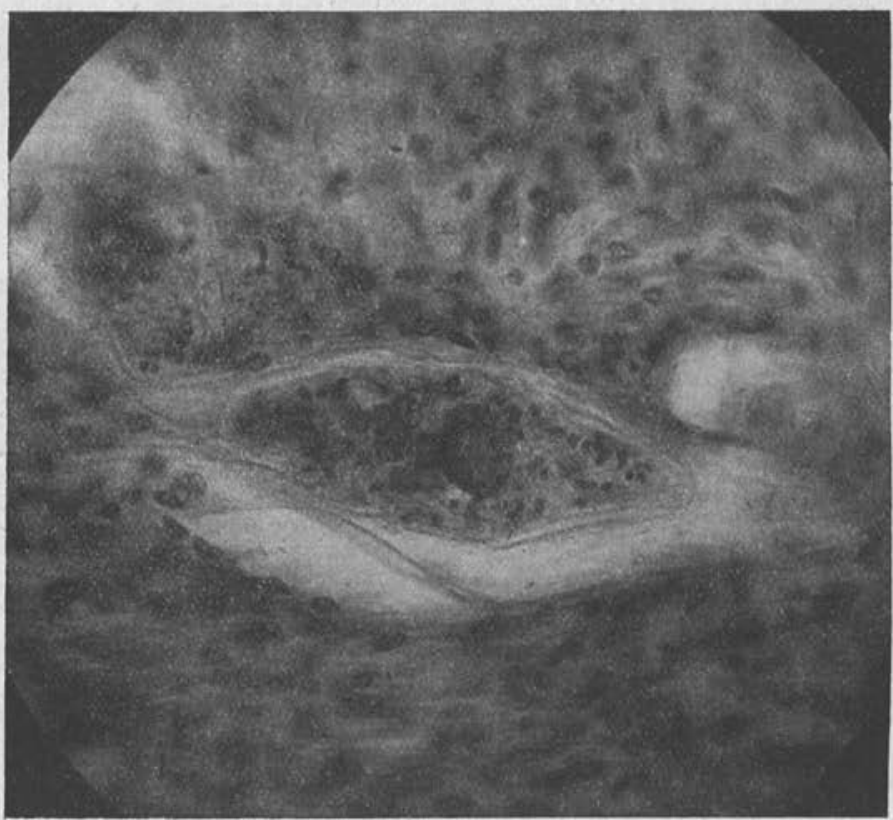

Fig. 10. - Microphotographie d'une coupe sagittale d'un œuf de Schistosoma bovis. La masse ronde placée à droite est considérée génếralement par les auteurs comme étant l'ébauche du système nerveux. On voit distinctement à gauche quatre cellules germinales qui deviendront des sporocystes fils.

de 161 à 233 œufs dans leur utérus ; il est juste d'ajouter que dans le même travail Faust et Meleney atténuent l'importance de leur hypothèse en disant que leurs constatations, concernant les œufs utérins, ne permettent pas d'établir une comparaison absolue entre Ia fécondité de $S$. japonicum et celle de $S$. mansoni.

Les expériences et les études statistiques que j'ai faites me permettent d'affirmer, d'une part que je mêtais trompé en parlant de la faible fécondité des femelles de $S$. hæmatobium et de $S$. mansoni en 1922, et d'autre part que la réserve formulée par Faust et Mele- 
ney était tout à fait justifiée. En effet, il résulte de mes études que, quel que soit le nombre d'œufs séjournant dans l'utérus des femelles, la ponte quotidienne est à peu près identique, elle semble même plus considérable chez $S$. mansoni, présentant habituellement un seul œuf, que chez $S$. hæmatobium présentant de 10 à 30 œufs et chez $S$. bovis, dont l'utérus peut renfermer dans des conditions expérimentales de 3 à 39 øufs.

La méthode utilisée pour apprécier la fécondité des femelles d'une espèce déterminée est très simple. Elle consiste à évaluer le nombre d'œufs trouvés dans le foie et dans l'ensemble de l'intestin et de diviser ce chiffre par le nombre de jours de ponte, puis par le nombre de femelles récoltées. Le résultat est évidemment assez approximatif puisque, d'une part, des œufs se rencontrant dans d'autres organes (rate, pancréas, mésentère, estomac, poumon) ne sont pas comptés, et que, d'autre part, quelques femelles peuvent échapper aux recherches, ce qui compense la première cause d'erreur. Cependant, comme les résultats obtenus sont très comparables entre eux, cette méthode d'appréciation est en somme assez exacte et permet de comparer la fécondité des diverses espèces.

Prenons le cas des souris $115 \mathrm{XI}$ et $116 \mathrm{XI}$, sacrifiées 39 jours après le premier bain infectieux renfermant des cercaires de $S$. mansoni et environ six jours après la première ponte des femelles de schistosomes qu'elles hébergent. La souris 115 XI présente 10 à 15 oufs par champ microscopique (obj. 2, oc. 1 Stiassnie) dans la pulpe de foie écrasée. Une évaluation volumétrique, combinée avec une évaluation par pesée, donne le chiffre de 29.200 œufs pour la masse totale du foie. Les 28 centimètres d'intestin grêle, qui montrent une moyenne de 1.000 oufs par centimètre, nous donnent le chiffre de 28.000 œufs et les 10 centimètres de gros intestin 5.000 , soit un total de 62.200 œufs. Ces œufs ont été pondus par 66 femelles en six jours environ, ce qui permet de dire que chaque femelle a pondu (63.200:66) 948 œufs, soit 157 par jour et 6,5 par heure environ.

En faisant un calcul identique pour les femelles hébergées par la souris $116 \mathrm{XI}$, nous trouvons un total de 60.800 œuf́s déposés en six jours également par 53 femelles, soit 1.146 œufs pour chacune d'elles pendant toute la durée de la ponte, 191 par jour et environ 8 par heure.

Dans le cas du Schistosoma bovis, je crois pouvoir admettre que Ia ponte ne commence pas avant le $60^{\circ}$ jour. En effet, la souris $140 \mathrm{XI}$, morte le $63^{\circ}$ jour, présentait quelques rares œufs dans le rectum et dans le foie et aucun dans les autres organes, bien que 14 femelles, dont 12 accouplées, aient été récoltées chez cet animal. 
Chez les sujets morts ou sacrifiés plus tard, par exemple chez les animaux $142,143,144,145$, morts respectivement $73,73,74$ et 75 jours après l'infection, tous les organes étaient fortement infectés par les œufs bien que les femelles récoltées aient été relativement peu nombreuses, 26 chez la souris 142 XI et $19 \mathrm{chez}$ la souris $143 \mathrm{XI}$.

Des calculs, effectués dans le cas de trois souris, mortes 79,87

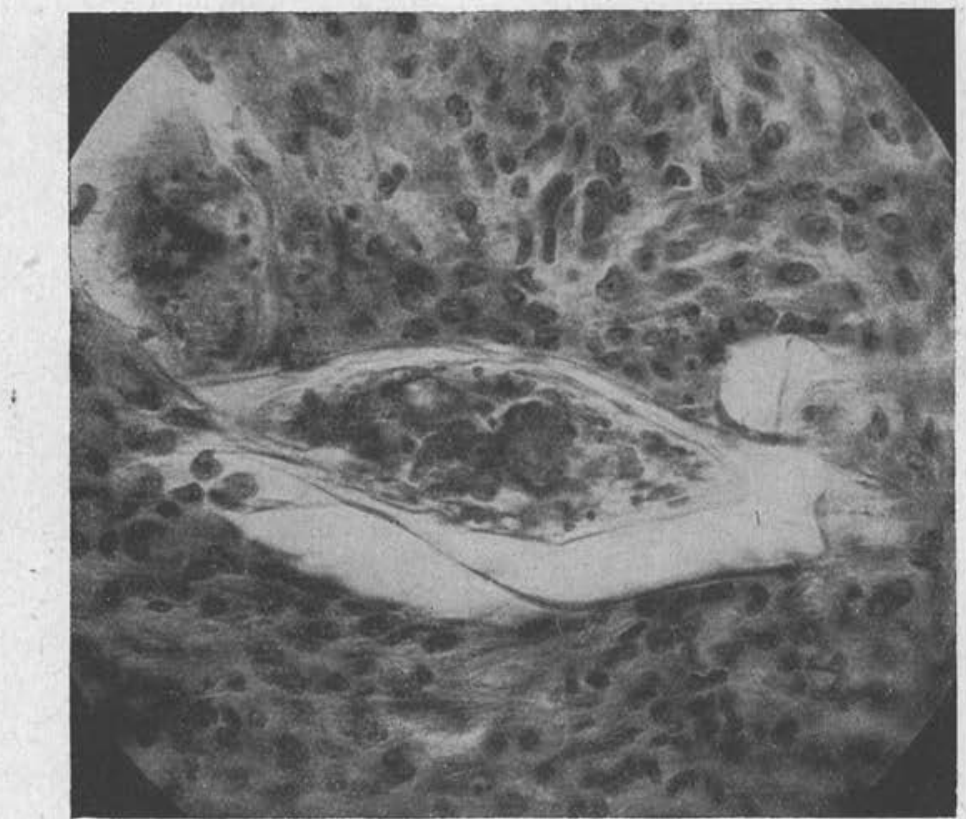

FIG. 11. - Microphotographie faite sur un autre plan de l'œuf déjà représenté sur la fig. 10. On voit l'ébauche nerveuse et à gauche plusieurs cellules mères de sporocystes fils.

et 95 jours après la première infection, donnent les résultats suivants : la souris $129 \mathrm{XI}$, sacrifiée agonisante le $79^{\circ}$ jour, montre un total d'environ 40.800 œufs pour 36 femelles ayant pondu probablement depuis le $60^{\circ}$ jour, ce qui donne une ponte quotidienne de 60 oufs et une ponte horaire de 2,5 œufs.

La souris $150 \mathrm{XI}$, morte le $87^{\circ}$ jour, renferme 16 femelles ayant déposé 48.000 œufs en 27 jours, soit une ponte de 111 œufs par jour et de 4,6 œufs à l'heure pour chaque femelle.

Enfin, la souris $155 \mathrm{XI}$, morte le $95^{\circ}$ jour, est parasitée par 32 femelles ayant dû pondre depuis 35 jours environ 25,5 œufs par 
jour, soit environ un par heure en admettant que la ponte soit égale pendant toute la durée de l'infection, ce qui reste à démontrer. J'ai cependant l'impression, d'après diverses données anatomo-pathologiques, que les femelles pondent davantage quand elles sont jeunes.

Les faits résumés ci-dessus montrent donc que les femelles de $S$. mansoni, n'ayant qu'un œuf utérin, sont plus fécondes que celles de $S$. bovis qui peuvent en présenter parfois 35 ou 39 . Je n'ai pas de statistique aussi précise à présenter pour les femelles de $S$. hæmatobium, mais, d'après les documents fragmentaires que j'ai pu trouver dans mes protocoles d'autopsies de souris, l'infection des organes par les œufs de ces vers est moins considérable que dans le cas du $S$. mansoni.

Miracidium. - L'évolution du Schistosoma bovis fera l'objet d'un mémoire particulier. Je signalerai brièvement ici que les œufs, mis en liberté par le broyage du foie ou des autres organes parasités, par le raclage de l'intestin ou par la dilution des déjections, éclosent très rapidement dans de l'eau à $20-25^{\circ}$ et à la lumière naturelle dans la journée ou le soir à la lumière électrique. Après 10 minutes de contact avec l'eau, les miracidium éclosent et circulent avec une grande rapidité comme ceux de $S$. hæmatobium et $S$. mansoni; ils possèdent comme ces derniers quatre flammes excrétrices vibratiles (1).

L'attraction miracidienne peut être étudiée quand les œufs sont abondants, ce qui arrive en utilisant le produit de broyage d'un foie. Malheureusement,' comme le liquide reste toujours trouble, il est difficile de suivre les miracidium qui s'approchent des Bullinus et qui semblent se fixer sur les parties visibles de leur corps. Les mollusques soumis à l'expérimentation semblent bien supporter l'infection; cependant, dans deux élevages, une mortalité assez considérable a été observée une douzaine de jours après le début de l'expérience.

Cercaires. - Chez les Bullinus infectés naturellement en Corse et émettant des cercaires, l'hépatopancréas était rempli de sporocystes à parois très minces qu'il était impossible d'isoler par la dissection, mais qu'il est facile d'étudier sur des coupes comme celles représentées en 1 et 2 , pl. 1 (2).

(1) L'appareil excréteur du miracidium de $S$. bovis, comme d'ailleurs celui du miracidium de $S$. hamatobium et $S$. mansoni, est très difficile à étudier. Malgré l'abondant matériel que je possède, il m'a été impossible jusqu'à présent de voir d'une façon nette les deux pores excréteurs latéraux signalés et représentés par Looss, Cort, Faust et Meleney.

(2) Jadresse mes bien sincères remerciements au Dr M. Langeron à qui je suis redevable des microphotographies jointes à ce travail. 
Les cercaires de $S$. bovis ressemblent beaucoup à celles de $S$. hæmatobium et de $S$. mansoni. Je donne dans le tableau ci-dessous les dimensions des trois espèces de cercaires obtenues expérimentalement par moi chez leurs hôtes respectifs :

\begin{tabular}{|c|c|c|c|c|c|c|c|c|c|c|}
\hline \multirow{4}{*}{ Corps...... $\left\{\begin{array}{l}\text { longueur... } \\
\text { largeur..... }\end{array}\right.$} & \multicolumn{4}{|c|}{ S. bovis (1) } & \multicolumn{3}{|c|}{ S.hamatobium (2) } & \multicolumn{3}{|c|}{ S. mansoni } \\
\hline & A. & в. & c. & D. & A. & B. & $\begin{array}{c}\text { C. } \\
\text { (profil) }\end{array}$ & A. (3) & B. (4) & $\begin{array}{l}\text { C. (8) } \\
\text { (profil) }\end{array}$ \\
\hline & 160 & 160 & 160 & 160 & 166 & 166 & 166 & 160 & 173 & 137 \\
\hline & 50 & 50 & 50 & 50 & 66 & 66 & 60 & 58 & 53 & 56 . \\
\hline Tronc caudal. longueur. & 220 & 240 & 240 & 230 & 223 & 237 & 200 & 230 & 215 & 200 \\
\hline 1ronc catuar. I largeur... & 30 & 30 & 30 & 30 & 35 & 31 & 35 & 33 & 33 & 32 \\
\hline Insertion du tronc caudal & 15 & 15 & 15 & 13 & 14 & 14 & 11 & 18 & 18 & 18 \\
\hline Branches, longueur. & 80 & 80 & 70 & 70 & 90 & 73 & 70 & 84 & 78 & 68 \\
\hline de la fourche / largeur (base) & 15 & 15 & 15 & 15 & 12 & 12 & 13 & 13 & 12 & 18 \\
\hline (longueur. & 55 & 55 & 55 & 55 & 56 & 56 & 56 & 56 & 68 & 49 \\
\hline largeu & 31 & 31 & 31 & 30 & 37 & 37 & 37 & 37 & 37 & 35 \\
\hline Ventouse ventrale. & 20 & 21 & 21 & 21 & 20 & 20 & 20 & 20 & 21 & 20 \\
\hline
\end{tabular}

Ces trois espèces de cercaires ont été tuées en extension par la chaleur dans des tubes à essais et conservées dans une solution de formol à 5 pour 100 , ce qui permet de les comparer d'une façon aussi précise que possible. Les fig. 12,13 et 14 représentent ces cercaires telles qu'elles se montrent après usage de la technique signalée ci-dessus.

Pour bien étudier la structure de ces larves, il faut s'adresser de préférence à celles qui sont encore dans l'hépatopancréas du mollusque, car elles vivent plus longtemps et se déplacent moins facilement entre lame et lamelle que celles évacuées spontanément. Il est possible, dans ces conditions, de constater l'existence de flammes vibratiles. Ces dernières sont probablement au nombre de dix : quatre de chaque còté du corps et deux à la racine de la queue. Cependant, comme il m'a été impossible de voir dix flammes à la fois sur la même cercaire et comme le nombre des exemplaires

(1) Cercaires d'un mélange provenant de 12 Bullinus infectés spontanément.

(2) Cercaires provenant d'un mélange obtenu avec plusieurs Bullinus infectés expérimentalement.

(3) Cercaires d'un seul Planorbis boissyi (Exemplaire 23).

(4) Cercaires d'un seul Planorbis boissyi (Exemplaire 19). 
favorables était très restreint, il est possible que leur nombre soit seulement de huit, comme chez les schistosomes parasites de l'homme, d'après Cort et Faust. Les canaux excréteurs sont difficiles à suivre, ils se réunissent à la partie postérieure du corps en un tronc commun qui traverse toute la queue, se bifurque an
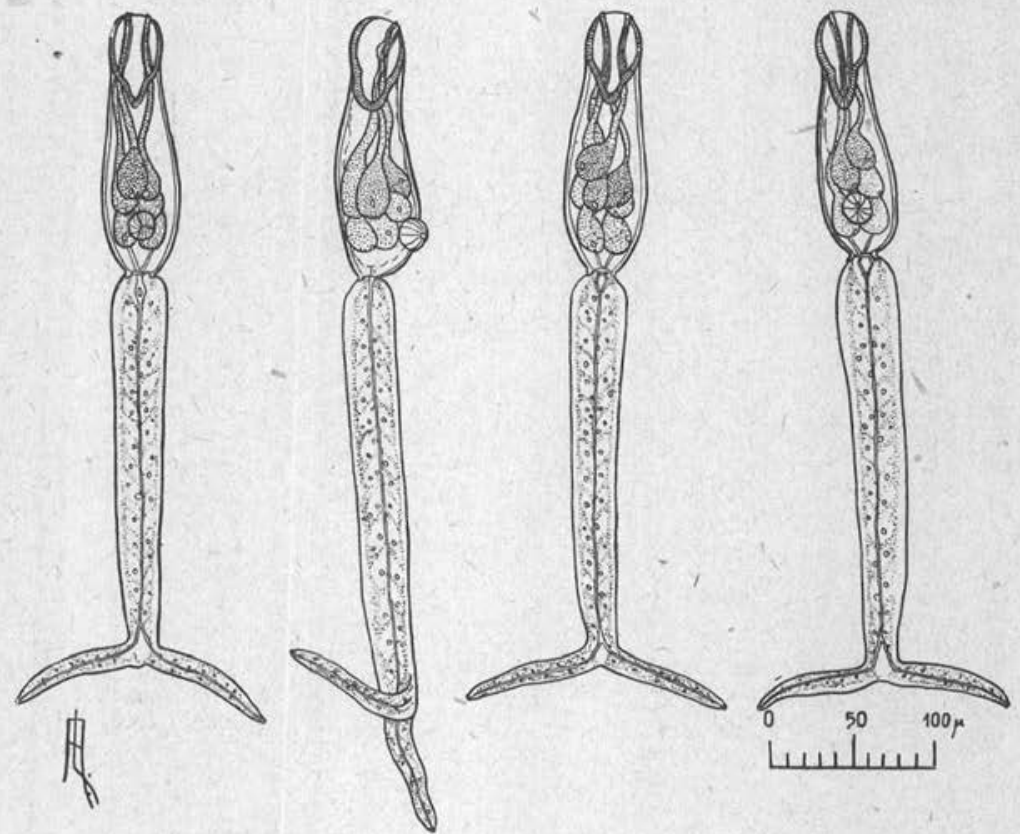

Fig. 12. - Furcocercaires de Schistosoma bovis tuées par la chaleur et conservées dans du formol à 5 pour cent. Il me semble impossible de voir davantage sur du matériel mort conservé dans ce fixateur. La description de cette cercaire sera faite ultérieurement, d'après du matériel frais et du matériel fixé, coupé et coloré par diverses méthodes.

niveau des branches de cette dernière qui présentent chacune à leur extrémité un pore excréteur.

La cercaire de $S$. bovis est entièrement recouverte de petites épines qui tapissent également l'intérieur de la ventouse ventrale. Dans la partie antérieure du corps, au niveau de la capsule, on observe 45 à 50 rangées longitudinales de petites épines dont la pointe est dirigée en arrière. La capsule musculaire antérieure est très nette et présente à peu près la même forme vue de face, de dos ou de profil. Les glandes céphaliques, divisées en deux groupes, un 
groupe antérieur hyalin et un groupe postérieur granuleux, semblent au nombre de dix ; leurs conduits aboutissent isolément, de chaque côté de l'extrémité antérieure, à de petites papilles au nombre de quatre ou einq. L'appareil digestif est constitué par un tube simple dépourvu de pharynx musculeux; il se termine par une bifurcation, formée de deux branches très courtes.

Comme les mollusques servant à mes études étaient réservés
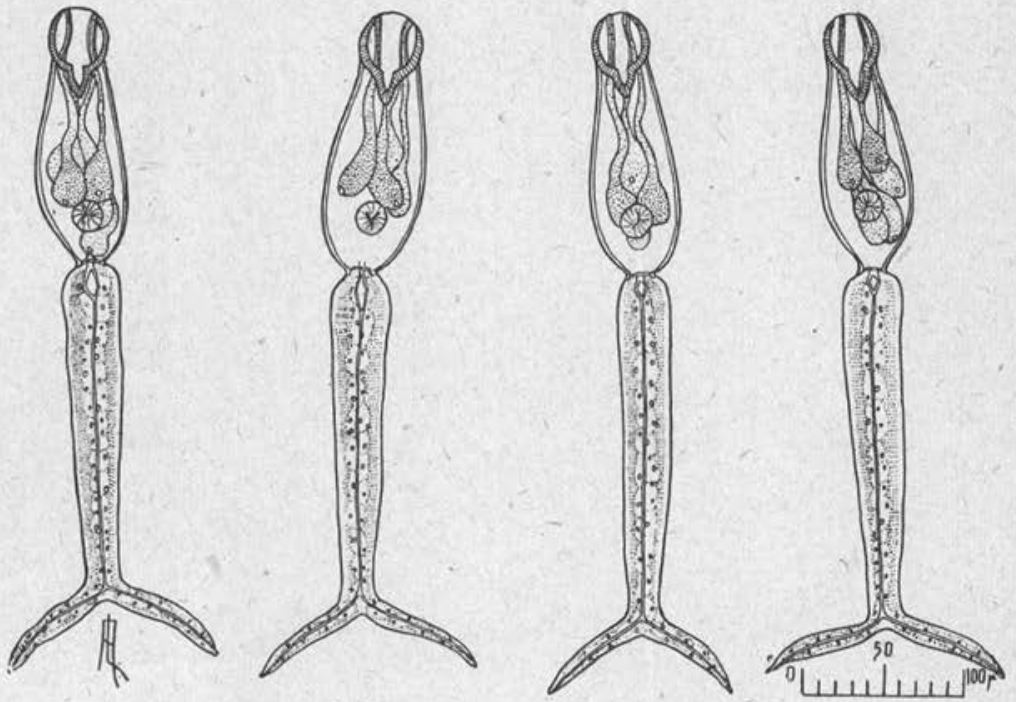

Fig. 13. - Furcocercaires de Schistosoma hrmatobium tuées par la chaleur et conservées dans du formol à 5 p. 100 comme dans le cas de Schistosoma bovis. Cette cercaire sera étudiée ultérieurement sur du matériel frais.

pour infecter divers animaux de laboratoire, je n'ai pas voulu en sacrifier un nombre suffisant pour compléter l'étude morphologique de la cercaire vivante qui sera faite plus tard.

La cercaire de $S$. bovis semble quitter le mollusque-hôte aussi bien le jour que la nuit à la température de $25^{\circ} \mathrm{C}$. Elle nage rapidement dans l'eau avec la queue généralement en avant, mais parfois en arrière. Elle peut également ramper en se fixant par ses ventouses et elle arpente le substratum sur lequel elle se trouve et duquel il est parfois difficile de la détacher, même à l'aide d'une pipette.

Après sa libération de l'hôte qui l'a engendrée, cette cercaire vient se reposer au-dessous de la surface de l'eau; elle enroule alors en crosse les deux branches de la fourche caudale. 
Les mollusques parasités étudiés par moi n’émettaient pas autant de cercaires que ceux hébergeant $S$. hæmatobium ou $S$. mansoni, tout au moins d'après mes expériences personnelles. Les plus infectés n'émettaient pas, du 20 août au 14 septembre, jour de la mort du dernier spécimen, plus de 500 à 600 cercaires par jour.

Il est vrai que les mêmes faits peuvent s'observer avec les autres
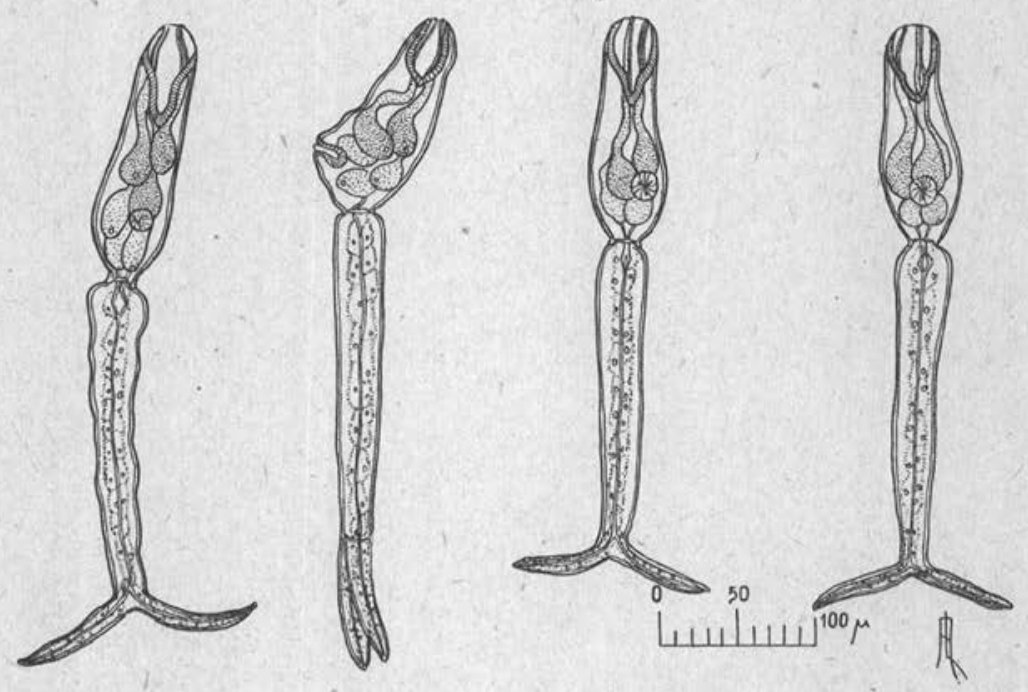

Fig. 14. - Furcocercaires de Schistosoma mansoni fixées par la clialeur et conservées dans du formol à 5 pour 100 . La structure glandulaire est très difficile à étudier sur de semblables animaux. Cette cercaire fera ultérieurement l'objet d'une êtude morphologique comparative avec celles de deux autres espèces figurées p. 40 et 41 .

schistosomes vers la fin de l'infection des mollusques. Or, comme les Bullinus étudiés avaient été récoltés déjà infectés, il a été impossible d'établir depuis quand ces animaux émettaient des cercaires. Si les expériences d'infection de mollusques neufs que je poursuis actuellement réussissent (1), j'espère pouvoir étudier d'une manière plus précise, ce très intéressant,phénomène. Les cercaires sortent par petits groupes de la cavité palléale du mollusque.

(1) Pendant l'impression de ce mémoire, j'ai constaté que les mollusques placés dans une étuve à $28^{\circ}$ s'étaient très facilement infectés. Dès le $21^{1}$ jour, j’ai vu une furcocercaire dans l'élevage 389 XI et le $39^{\circ}$ jour, dans ce même élevage, sur 44 Bullinus encore vivants, 38 étaient positifs et 6 négatifs. Dans l'élevage $402 \mathrm{XI}$, le 32 e jour, sur 96 Bullinus, 73 étaient positifs. Enfin dans l'élevage $415 \mathrm{XI}$, il y avait le $28^{\circ}$ jour 16 mollusques infectés et 10 négatifs. 
La longévité des cercaires est faible à une température de $25^{\circ} \mathrm{C}$; il est rare d'en trouver encore de vivantes après 48 heures.

Rôle pathogène. - Les animaux de laboratoire, infectés par un trop grand nombre de cercaires, succombent avant la maturité des vers avec des embolies parasitaires dans les organes, en particulier dans le foie (fig. 3 , pl. 1). Les animaux moins infectés, comme c'est le

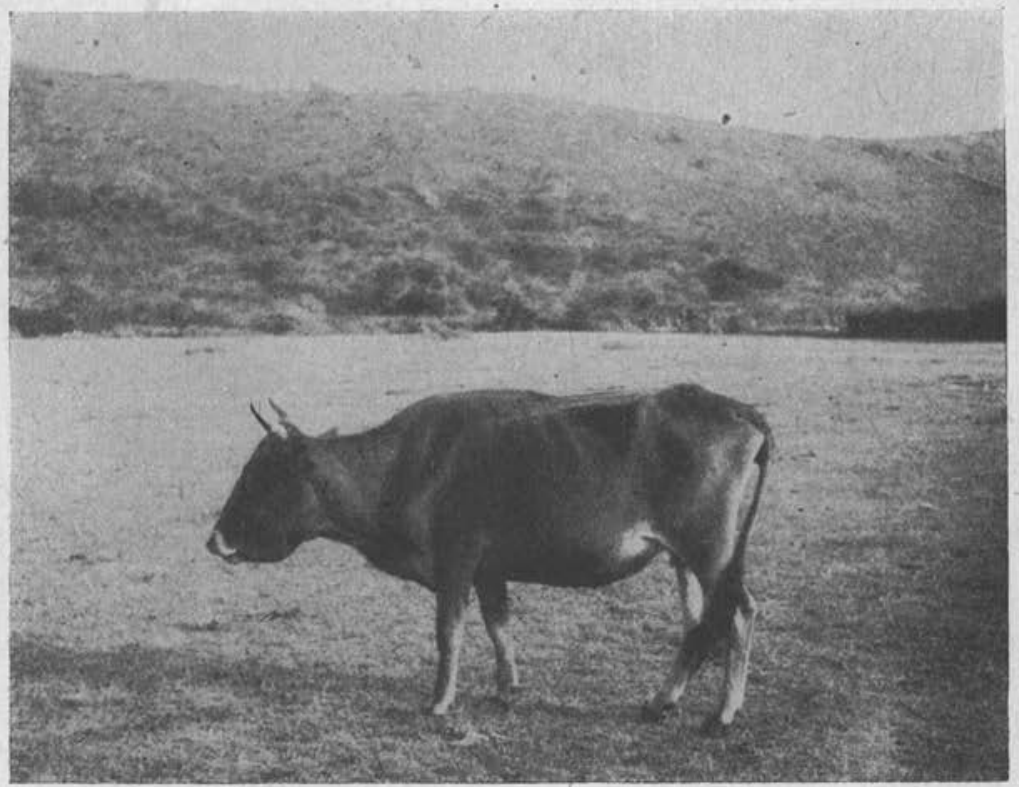

Fig. 15. - Vache corse de la région de Monacia, âgée de 4 ou 5 ans, montrant un œdème intermaxillaire ( ( bouteille $)$ ) très accentuée. Cet animal présentait de nombreux œufs de Paramphistomum cervi dans ses selles.

cas pour les diverses souris signalées sur le tableau de la page 32 , meurent au moment où les œufs produisent dans le foie une cirrhose extrêmement intense. Les lésîons hépatiques sont peu marquées chez les animaux infectés exclusivement par un sexe, fait que j'ai pu observer déjà chez deux souris ne présentant que des Schistosoma hæmatobium mâles et que je viens d'observer chez une souris infectée par les cercaires de $S$. bovis provenant d'un seul mollusque. D'autre part, la présence de parasites des deux sexes et de leurs oufs dans les organes semble empêcher la croissance des souris infectées, encore plus que dans 
le cas des autres espèces de schistosomes (S. hæmatobium et $S$. mansoni). L'infection pure par des mâles de $S$. bovis ne semble pas empêcher la croissance ; c'est ainsi que la souris $197 \mathrm{XI}$, sacrifiée étant bien portante 89 jours après l'infection, pesait 27,975 grammes et que les autres souris, infectées également par un seul mollusque, pesaient respectivement : $28,25,25,5,28,21$, 21 et 27,5 grammes, alors que des souris témoins de même âge,

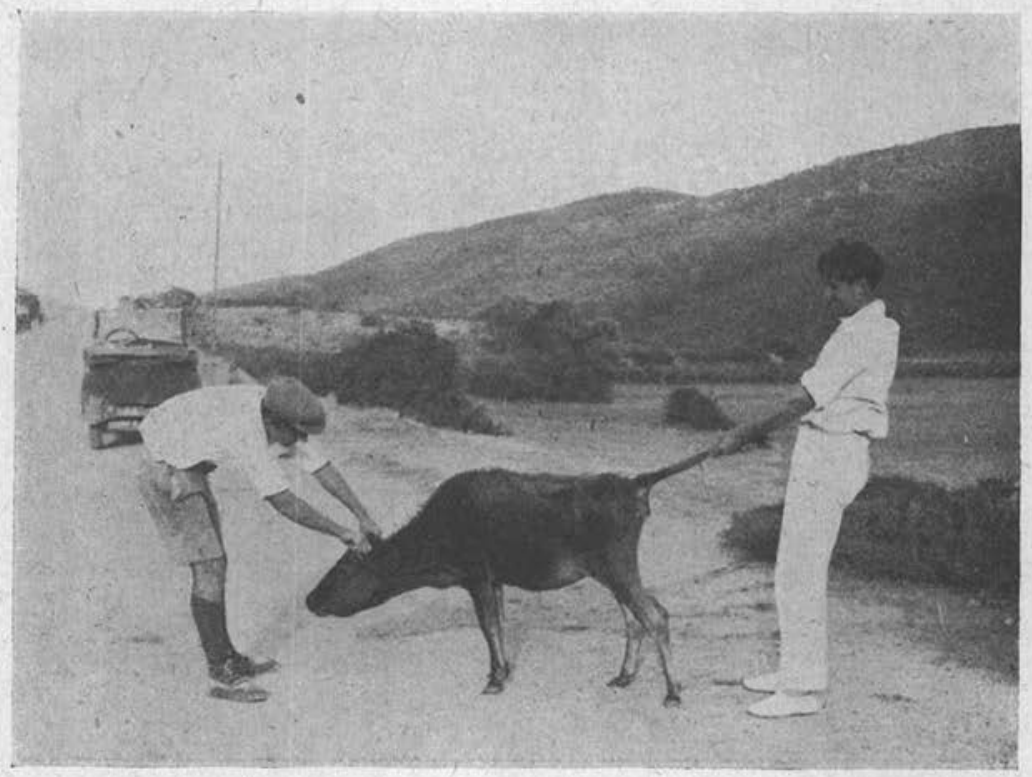

Fig. 16. - Veau femelle de 10 à 12 mois, déjà atteint de cachexie et montrant de l'cedème intermaxillaire. Ses selles renfermaient des œufs de Paramphistomum cervi.

au nombre de 19 , infectées par des $S$. bovis des deux sexes, pesaient de 10,732 à 22,480 grammes (voir tableau, p. 32). Les souris témoins neuves du même âge pèsent de 25 à 30 grammes.

Chez les ruminants, je n'ai pu faire encore aucune expérience, mais il est probable qu'une infection intense doit provoquer des troubles sérieux de leur santé. C'est probablement ce qui se produit à Monacia, où il est impossible d'entretenir des troupeaux de moutons et de chèvres dans des pâturages d'excellente qualité et où les bovins eux-mêmes présentent un état cachectique marqué dans bien des cas, même chez des animaux àgés (fig. 17). Il est vrai que les trois animaux du troupeau photographié, dont j'ai pu me pro- 
curer des selles, hébergeaient un nombre plus ou moins grand de Paramphistomum cervi, mais par contre aucune des deux douves du foie ( $F$. hepatica et $D$. lanceatum) assez répandues en Corse. Malgré l'absence d'œufs de Schistosoma bovis dans les selles examinées, je pense que la cachexie observée chez les animaux de Monacia

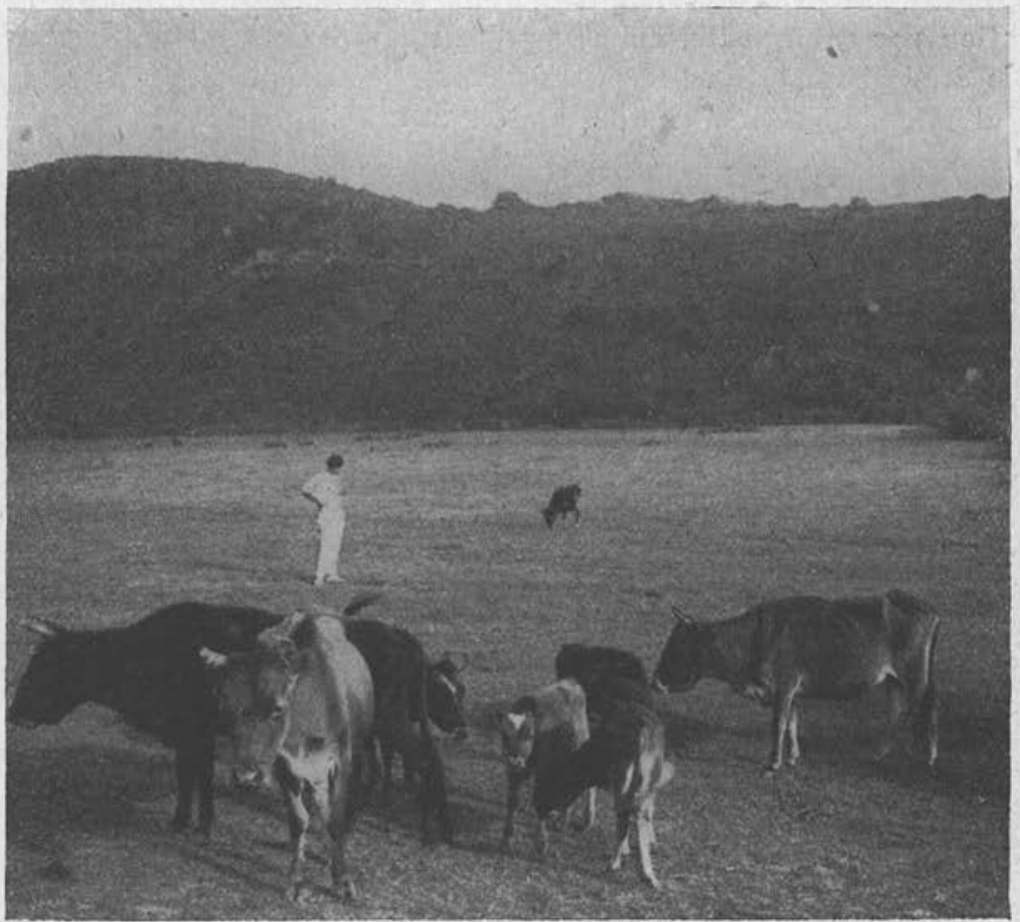

Fig. 17. - Troupeau de Monacia s'abreuvant dans les mares à Bullinus; trois vaches et un veau présentaient un fort œdème intermaxillaire et une grande maigreur, malgré l'excellente qualité et l'abondance des pâturages.

est due en grande partie à ces vers, d'autant plus que Liston et Soparkar (1917) ont observé une cachexie accompagnée d'œdème intermaxillaire (bouteille) chez une chèvre infectée expérimentalement par eux avec Schistosoma spindalis.

En ce qui concerne l'homme, je crois, malgré les observations de Stephens et Christophers (1905), concernant un Hindou ayant habité le Natal, de Cawston $(1925 b)$ dans ce dernier pays et de Mahfuz (1927) en Egypte, que la réalité de cette infection reste à 
démontrer, les œufs de $S$. hæmatobium trouvés dans l'urine étant assez polymorphes et pouvant prêter à confusion. Un fait certain, c'est qu'ayant manipulé sans précaution des milliers de Bullinus avant de connaître leur infection et même après, je pense ne pas héberger de $S$. bovis, à en juger tout au moins par les analyses négatives que j'ai effectuées.

Il est d'ailleurs possible que certains sujets soient sensibles à l'infection par ce parasite, mais ce point ne pourra être établi que par une étude complète, comprenant l'infection d'hòtes intermédiaires et d'hôtes définitifs, des geufs attribués à ce ver et trouvés dans l'urine ou dans les déjections de l'homme. Si ce dernier était sensible, il y a bien longtemps que dans les pays où la bilharziose bovine ou ovine est répandue (Sicile, Sardaigne, Egypte) et où l'examen des selles est pratiqué souvent sur des milliers d'individus, sa présence aurait été établie.

\section{RÉSUMÉ}

$1^{\circ}$ L'existence en Corse de Bullinus contortus, infecté par une furcocercaire de schistosome ayant produit expérimentalement, chez la souris, le hérisson et le cobaye, seuls animaux dont la réceptivité a été étudiée, la forme adulte du Schistosoma bovis, permet d'affirmer la présence de ce ver en Corse.

$2^{\circ}$ Dans les gîtes de Monacia, où ont été trouvés des Bullinus contortus infectés, le pourcentage, les 15 et 20 août 1929, était environ de 1 (1). Mais en établissant le rapport entre les mollusques parasités et l'ensemble de ceux récoltés dans les diverses mares de cette localité, le pourcentage s'abaisse aux environs de 0,5.

$3^{\circ}$ Aucun Bullinus des autres régions de Corse, examinés à la même saison, en 1927 et 1928 , ne présentait de furcocercaires. La distribution de $S$. bovis en Corse est donc très limitée.

$4^{\circ}$ Les mollusques trouvés infectés le 15 et le 20 août présentaient une longueur variant de 4 à 10 millimètres.

$5^{\circ}$ Dans les conditions expérimentales, chez la souris, quand les deux sexes de schistosomes coexistent, les adultes se rencontrent en plus grand nombre dans le foie que dans l'ensemble des autres organes. Chez une souris infectée par un seul mollusque et n'ayant que des mâles, ces derniers étaient beaucoup plus nombreux dans le mésentère que dans le foie.

$6^{\circ}$ Le nombre de schistosomes rencontrés chez les animaux d'expérience peut être considérable. C'est ainsi que des souris

(1) Expérimentalement, à la température constante de $28^{\circ} \mathrm{C}$, ce mollusque s'infecte en trois semaines, dans une proportion dépassant parfois $80 \mathrm{p} .100$. 
pesant de 11 à 22 grammes peuvent héberger de 14 à 120 mâles et de 14 à 36 femelles.

$7^{\circ}$ Les femelles pondent des œufs vers le $60^{\circ}$ jour et les œufs déposés dans les tissus semblent présenter un miracidium bien développé deux ou trois jours plus tard, bien que des cultures d'œufs n'aient pas permis de préciser ce point.

$8^{\circ}$ Les femelles de $S$. bovis, dont l'utérus peut renfermer jusqu'à 39 œufs à la fois, ont une moins grande fécondité que celles de $S$. mansoni qui n'en présentent qu'un, ainsi qu'il résulte de calculs faits chez des souris autopsiées ou sacrifiées à des dates diverses. Il semble, en effet, que, tout au moins pendant les premières semaines qui suivent leur fécondation, les femelles de $S$. mansoni pondent environ de 157 à 191 œufs par jour, soit environ 6,5 à 8 par heure.

Les femelles de $S$. bovis pondent de 25,5 à 111 oufs par jour, ce qui donne respectivement pour trois cas étudiés 60,111 et 25,5 œufs pondus par jour et 2,5, 4,6 et 1 par heure.

$9^{\circ}$ Malgré la ponte fréquente des femelles et les nombreuses souris examinées, l'auteur n'a jamais pu saisir, pour les trois espèces de schistosomes étudiées par lui (S. hæmatobium, S. mansoni, S. bovis), l'acte de la ponte dans les capillaires veineux où leurs œufs abondent, ce qui lui permet d'admettre que nous en sommes réduit aux hypothèses, en ce qui concerne le dépòt des œufs dans les organes.

$10^{\circ}$ Les dimensions des œufs utérins varient, chez les femelles obtenues expérimentalement, entre $63 / 20 \mu$ et $175 / 50 \mu$; les dimensions habituelles sont de $160 / 40 \mu$.

Les œufs mûrs renfermant un miracidium sont plus grands, ils peuvent atteindre $280 / 90 \mu$; les dimensions moyennes sont d'environ $220 / 60 \mu$.

11. Le miracidium, pourvu de quatre flammes vibratiles, éclôt en quelques minutes dans l'eau à $25^{\circ} \mathrm{C}$. et à la lumière naturelle ou artificielle.

$12^{\circ}$ Les cercaires, dépourvues de pharynx, ont des dimensions moyennes identiques à celles des cercaires de Schistosoma hæmatobium et $S$. mansoni, étudiées comparativement avec du matériel préparé suivant la même technique, consistant à tuer les animaux fixés en extension par la chaleur et à les conserver dans du formol à 5 pour 100 .

L'anatomie interne n'a pu être étudiée complètement faute de matériel. L'étude de cette cereaire fera l'objet d'un travail ultérieur.

$13^{\circ}$ La mort des mammifères infectés est déterminée soit par la présence de vers nombreux, même quand ces derniers sont jeunes, 
soit, surtout, par les œufs déposés dans les organes et produisant des lésions diverses incompatibles avec la vie. Chez des souris infectées avec des vers d'un seul sexe, même en nombre assez considérable, la santé n'est pas altérée et le foie semble normal à l'autopsie, bien qu'il présente cependant un certain nombre de lésions relativement discrètes.

$14^{\circ}$ La réalité de l'infection de l'homme par Schistosoma bovis, signalée ou admise par divers auteurs, reste à démontrer.

\section{BIBLIOGRAPHIE}

Alceo (G.). - La Bilharzia crassa negli ovini Sardi. La Clinica Vet., X̨XXXIX, 1926 , p. $78-81$.

Askar (M.). - Bilharziosis in Sudanese and Egyptian callle (1).

Barbagallo (P.). - Contributo allo studio della Bilharzia crassa in Sicilia. Arch. Parasil., II, 1899, p. 277-285.

Bayuss (H.-A.). - A manual of helminthology medical and velerinary. Baillière, Tindall and Cox, Londres, 1929, p. 49.

Bertolini (G.). - Osservazioni sulla Bilharzia crassa (Schistosomum bovis) e sulle alterazioni da essa prodotte. La Clinica Vet., 1908.

Bomford: - Note on eggs of Distoma (Bilharzia) hamalobium found in transport cattle. Scientific Memoirs by Med. Officers in India, Part. II, 1886, p. 53-55, et Quart. Journ. Vet. Science in India, V, 1887, p. 259.

British East Africa. - Report of the Department of Agriculture. Report of the Veterinary Department, Nairobi, 1911-1912.

Brumpt (E.). - La bilharziose au Maroc. Répartition du Bullinus contortus et du Planorbis metidjensis. Etude épidémiologique comparée du foyer tunisien de Gafsa et du foyer marocain de Marrakech. Bull. Soc. path. exot., XV, 1922 , p. 632.

- Précis de parasitologie, 3e édit., Paris, Masson, 1922, p. 375-388.

- Cycle évolutif du Schistosoma bovis ( = Bilharzia crassa). Infection spontanée du Bullinus contorlus en Corse. C. R. Acad. sc., CLXXXIX, 18 nov. 1929 , p. $879-881$. (Note préliminaire).

Cawston (F.-G.). - The Cercarize of the Natal. Journ. of Trop. Med. and. Hyg., XIX, 1916, p. 201-202.

- The Cercaria of the Transvaal. Parasilology, XI, 1918-1919, p. 93- -7 .

- a. Some Infecions due to fresh-water snails and their eradication. Journ. of Trop. Med. and Hyg., XXIII, 1920, p. 274-276.

- b. Schistosomes of man and beast in Natal. South Afric. Med. Rec., XVIII, 1920 , p. 264.

a. Bilharzia infected snails and their employement as antigen (Correspondance). Lancet, 1921 , p. 250.

b. Three schistosomes in Natal which possibly attack man. Journ. of Trop. Med. and Hyg., XXIV, 1921, p. 242.

The experimental infection of some animals with Cercaria from fresh-water

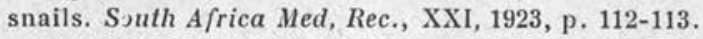

(1) Brochure, sans date, imprimée probablement en 1912 par le service vétéridu Caire, lue au laboratoire de Parasitologie de la Faculté de Médecine du Caire du Prof. M. Khalil. 
Some observations on the source of Bilharzia infection and the combined treatment of a case in Natal. Journ. of Trop. Med. and Hyg., XXVII, 1924, p. 283-284.

The identity of the rarer Schistosomes of man and their intermediary hosts. Ann. of Trop. Med. and Parasit., XIX, 1925, p. 215-218.

Environmental influences which favour the development and spread of the Bilharzia parasite in the South Africa. Food supply of Physopsis. Journ. of Trop. Med. and Hyg., XXIX, 1926, p. 89-91.

Chandeer (A.-C.). - A new Schistosome Infection of man, with notes on other human fluke infections in India, Indian Journ. of Med. Res., XIV, 1926, p. 179-181.

Chesterman (C.). - Note sur la bilharziose dans la région de Stanleyville (Congo belge). Ann. Soc. belge Med, Trop., II, 1923, p. 73-75.

Christophers (S.-R.) and Strphens (J.-W.-W.). - Note on a peculiar Schistosome egg. Journ. of Trop. Med., VIII, 1905, p. 259 et Brit. Med. Journ., II, 1905, p. 1289.

Cont (W.-W.). - 17. The Cercaria of the japanese blood fluke, Schistosoma japonicum Katsurada. 18. Notes on the eggs and miracidia of the human Schistosomes. University of California publications in Zoology, XVIII, 1914, p. 485-507, 3 fig. et p. 509-519, 7 fig.

FAust (E.-C.). - Notes on South African cercariæ. Journ. of Parasit., V, 1919, p. 164-175.

- Criteria for the differentiation of Schistosome larvæ. Journ. of Parasit., VI, 1920, p. 192-194.

A survey of Cawston's species of South African cercariæ. Parasitology, XII, 1920, p. 211-216.

Observations on South African larval trematodes. Parasitology, XVIII, 1926, p. 111.

- Human helminthology. Lea et Fibiger, edit. Philadelphie, 1929, p. 101.

Faust (E.-C.) et Meleney (H.-E.). - Studies on Schistosomiasis japonica. Amer. Journ. of Hyg., Monographic series, no 3, mars 1924, p. 182.

Füllebonn (F.). - Ueber Cercaria armata und Mücken larven. Arch. f. Sch. $u$. Trop. Hgg., XXVI, 1922, p. 78.

Grassi (B.). - Studi di un zoologo sulla malaria, Rome, 1900.

Grassi (B.) et Rovelli (G.). - La Bilharzia in Sicilia. Atti R. Accad. d. Lincei, LV, 1888 , p. 729.

KhaliL (M.). - On the morphology of Schistosoma bovis. Journ. of Helminthology, II, 1924, p. 81-86.

LEIPER (R.-T.). - Report on the results of the Bilharzia mission in Egypt. Journ. Roy. Army. Med. Corps, XXVII, 1915, p. 171-190.

Leuckart (R.). - Die Parasiten des Menschen, etc., II, 1886-1901, p. 464-534.

Liston (W.-G.) et Soparkar (M.-B.). - Bilharziosis among animals in India. The life cycle of Schistosomum spindalis. Ind. Journ. of Med. Res., V, 1918, p. $567-588$.

Looss (A.). - Schistosomum japonicum Katsurada, eine neue asiatische Bilharzia des Menschen. Centrlbl. f. Bakt. Orig., XXXIX, 1905, p. 280.

- What is Schistosomum mansoni Sambon, 1907. Ann. of Trop. Med. and Parasit., II, 1908, p. 153.

Mahfuz (A.-H.). - Un cas d'infection de l'homme par Schistosoma bovis. Egyptian Med. Rev., 1927, avril, p. 301-306 (en arabe).

Marotel (G.). - Existence de la bilharziose bovine en France. Recueil de Méd. Vét., LXXXV, 1908, p.119-122, 2 fig.

Annales de Parasitologie, $\mathrm{T}$. VIII, $\mathrm{x}^{\circ} 1 .-1^{\mathrm{er}}$ janvier 1930. 
Montgomery (R.-E.). - Observations on bilharziosis among animals in India. Ind. Journ. Trop. Vet. Sc., I, 1906, p. 15-46 et 138-174.

Price (E. -W.). - A synopsis of the trematode family Sclistosomidæ with description of new genera and species. Proc. U. S. National Mus., LXXV, 1929, p. 1-39, pl. 1-15.

Railliet (A.). - La bilharziose du bœuf en Annam. C. R. Soc. Biol., LI, 1899, p. 787.

SANFelice (F.) et LoI (L.). - Ueber das Vorkommen von Bilharzia crassa Sonsino in der Leber von Rindern in Sardinia. Centr. f. Bakt. Abt. I, XX, 1896, p. 305 .

- De alcune infezioni del bestiame studiate in Sardegna nel quadriennis 1892-96. Instituto d'Igiene della R. Universita di Cagliari, 1897.

Sewell. (R.-B.-S.). - Cercariz indica. The Indian Journ. of Med. Res., X suppl. number, juin 1922, p. 243.

Sonsino (P.). - Intorno ad un nuovo parassita del Bue (Bilharzia bovis) Rend. del Acc. di Scienze fis. e matem. di Napoli, XV, 1876, p. 84.

Soparkar (M.-B.). - A trematode parasite of Anopheline mosquitos. Ind. Journ. Med. Res., V, 1917, p. 512.

Werblunsky (S.). - Les hôtes intermédiaires de Schistosoma hæmatobium. Thèse Fac. Méd. Paris, 1928.

Station antipaludique de Porto-Vecchio (Fondation Rockefeller) et Laboraloire de Parasitologie de la Faculté de Médecine de Paris.

\section{Explication des Planches I et II}

\section{Planche I}

Fı́. 1. - Coupe de l'hépato-pancréas d'un Bullinus contortus (№ 45 XI) parásité dans des conditions naturelles par Schistosoma bovis. On distingue nettement au centre une furcocercaire entière .

FIG. 2. - Coupe dans le même organe montrant des cercaires dans les sporocystes fils à parois extrêmement minces et fragiles.

FIG. 3. - Coupe d'une veine du foie du hérisson $61 \mathrm{XI}$, mort de son infection le $37^{\circ}$ jour ; très nombreux vers déjà accouplés.

FIG. 4. - Coupe de la veine splénique du même hérisson, au point où elle aborde la rate. Comme les veines du foie, elle est remplie de vers jeunes mais déjà accouplés.

\section{Planche II}

FIg. 5. - Schistosome accouplé dans une petite veine (?) de la rate du hérisson $61 \mathrm{XI}$, mort d'une infection trop intense 37 jours après le début de l'expérience.

FIG. 6. - Foie d'une souris (144 XI) morte le $74^{\text {e }}$ jour de son infection. La coupe montre à sa partie supérieure un pseudo-tubercule renfermant deux œufs et à sa partie inférieure des Schistosoma bovis accouplés dans une veine.

FIG. 7. - Foie de la souris 151 XI, morte de 89 e jour; fragment écrasé montrant de grands œufs mûrs, transparents, avec miracidium, quelques œufs non mûrs ou dégénérés en bas et à droite et d'abondants dépôts de pigment noir (hémozoïne).

FIg. 8. - Eufs mûrs (les grands) et non mûrs de Schistosoma bovis dans le cæcum d'une souris (151 XI) morte le 89 e jour. Fait curieux le pigment noir ne semble jamais se rencontrer dans la paroi intestinale alors qu'il est toujours abondant dans le foie du même animal. 
ANNALES DE PARASITOLOGIE

T. VIII, No 1, 1er Janvier 1930.
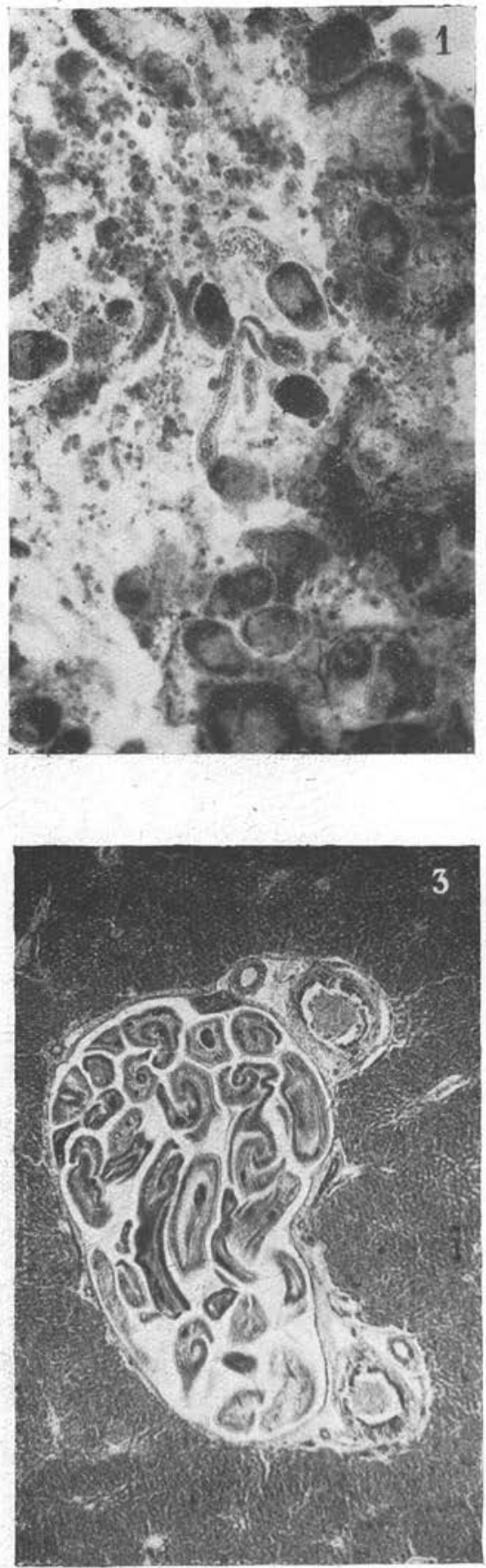

Planche I.

(Mèmoire Brumpt).
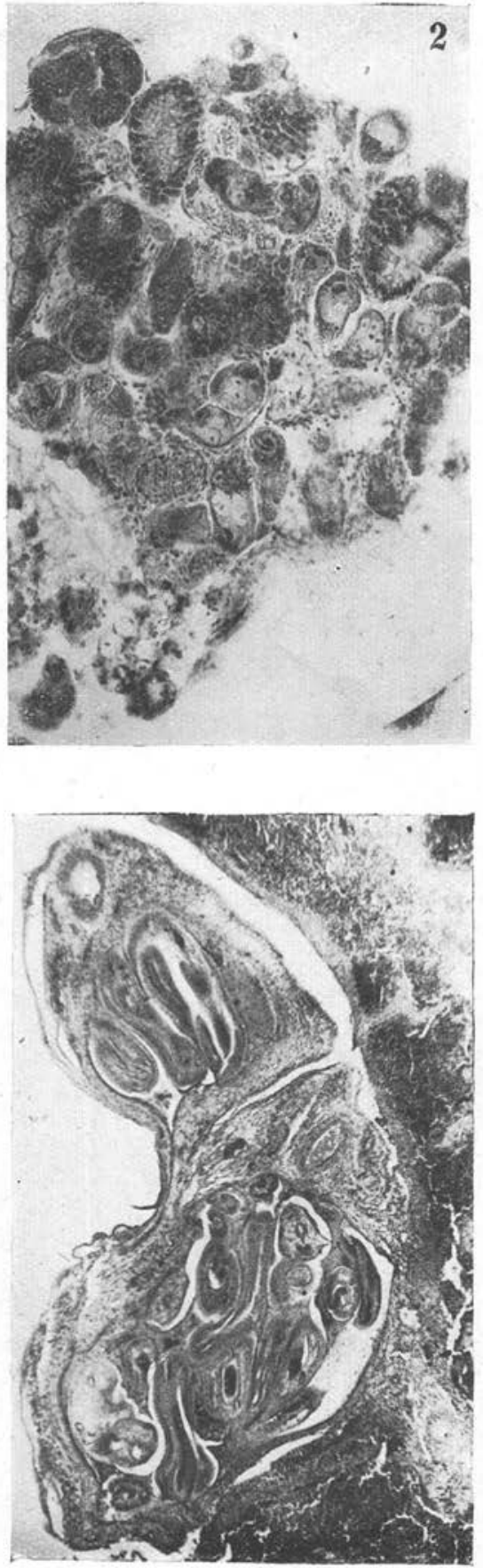

Masson et Cia, Editeurs. 

ANNALES DE PARASITOLOGIE

T. VIII, No 1, 1er Janvier 1930.
Planche II.

(Mémoire Brusipt).
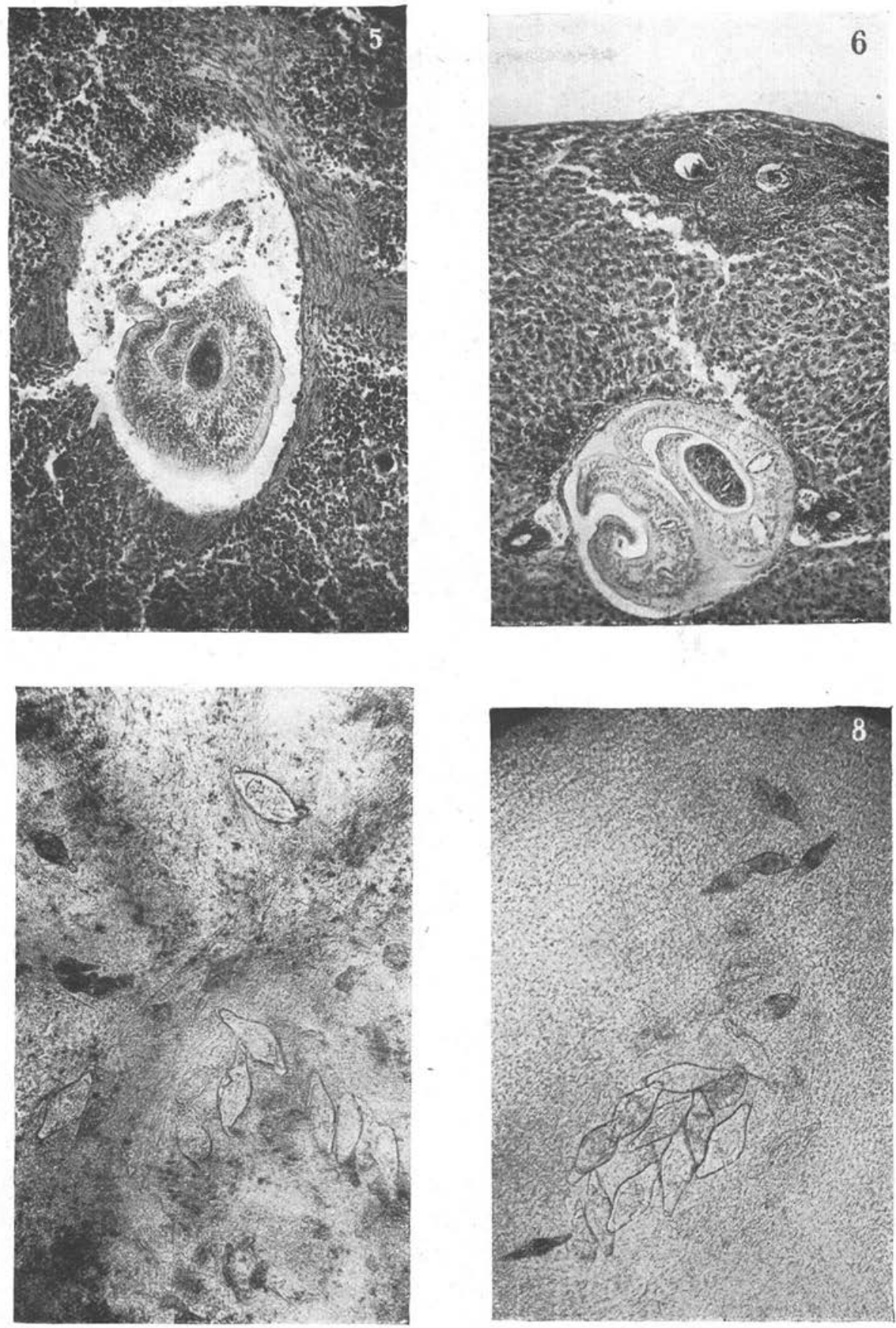

Masson et Cic, Editedis. 